\title{
Genome-wide identification of histone H2A and histone variant H2A.Z-interacting proteins by bPPI-seq
}

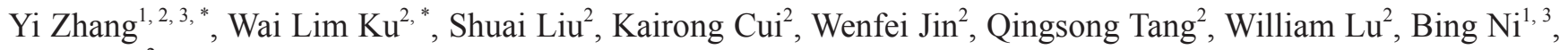
Keji Zhao ${ }^{2}$

${ }^{1}$ Institute of Immunology PLA, Third Military Medical University, Chongqing 400038, China; ${ }^{2}$ Systems Biology Center, Division of Intramural Research, NHLBI, NIH, Bethesda, MD 20892, USA, ${ }^{3}$ Department of Pathophysiology and High Altitude Pathology, Third Military Medical University, Chongqing 400038, China

$\mathrm{H} 2 \mathrm{~A}$ is a nucleosome core subunit involved in organizing DNA into a chromatin structure that is often inaccessible to regulatory enzymes. Replacement of $\mathrm{H} 2 \mathrm{~A}$ by its variant $\mathrm{H} 2 \mathrm{~A} . \mathrm{Z}$ renders chromatin accessible at enhancers and promoters. However, it remains unclear how H2A.Z functions so differently from canonical H2A. Here we report the genome-wide identification of proteins that directly interact with $\mathrm{H} 2 \mathrm{~A}$ and $\mathrm{H} 2 \mathrm{~A} . \mathrm{Z}$ in vivo using a novel strategy, bPPI-seq. We show that bPPI-seq is a sensitive and robust technique to identify protein-protein interactions in vivo. Our data indicate that H2A.Z-interacting proteins and H2A-interacting proteins participate in distinct biological processes. In contrast to $\mathrm{H} 2 \mathrm{~A}$-interacting proteins, the $\mathrm{H} 2 \mathrm{~A} . \mathrm{Z}$-interacting proteins are involved in transcriptional regulation. We found that the transcription factor Osr1 interacts with H2A.Z both in vitro and in vivo. It also mediates H2A.Z incorporation to a large number of target sites and regulates gene expression. Our data indicate that bPPI-seq can be widely applied to identify genome-wide interacting proteins under physiological conditions.

Keywords: bPPI-seq; protein-protein interactions; H2A; H2A.Z; Osr1

Cell Research (2017) 27:1258-1274. doi:10.1038/cr.2017.112; published online 1 September 2017

\section{Introduction}

In eukaryotic nuclei, nucleosomes, the building block of chromatin, are formed by wrapping $146 \mathrm{bp}$ of DNA around a histone octamer consisting two copies each of $\mathrm{H} 2 \mathrm{~A}, \mathrm{H} 2 \mathrm{~B}, \mathrm{H} 3$ and $\mathrm{H} 4$. While the vast majority of nucleosomes are composed of canonical core histones, a minor fraction of them are associated with histone variants. H2A.Z-containing nucleosomes are involved in multiple biological processes [1-3]. In contrast to H2A nucleosomes, H2A.Z-containing nucleosomes are specifically localized to transcriptional regulatory regions such as promoters and enhancers [4-9]. While H2A nucleosomes are generally involved in packaging DNA and condensation of chromatin, incorporation of H2A.Z facilitates tar-

\footnotetext{
*These two authors contributed equally to this work.

Correspondence: Keji Zhao, Bing Ni

${ }^{a}$ E-mail: zhaok@nhlbi.nih.gov

${ }^{\mathrm{b}}$ E-mail: nibingxi@126.com

Received 7 June 2017; revised 18 June 2017; accepted 22 June 2017; published online 1 September 2017
}

geting of transcription factors and chromatin regulators by maintaining chromatin accessibility [8-12]. Although crystallographic and biochemical studies revealed structural differences between H2A and H2A.Z nucleosomes [13], the underlying basis of the drastic differences in their function and the mechanisms that target H2A.Z to specific chromatin regions remains unclear.

Several studies have reported identification of proteins that co-purify with H2A.Z nucleosomes using affinity purification followed by mass spectrometry analysis (APMS). A number of proteins are found to co-purify with H2A.Z [14-16]. In particular, ANP32E was identified as an $\mathrm{H} 2 \mathrm{~A}$.Z-specific chaperone that mediates deposition of H2A.Z on chromatin [16, 17]. INO80 was identified as an H2A.Z-specific remodeler to remove H2A.Z for promoting homologous recombination in human cells [18]. While these studies identified some interesting H2A.Z-interacting proteins that may be involved in the downstream function of H2A.Z, it remains unclear how H2A.Z finds its specific target sites on chromatin. Thus more comprehensive knowledge of interacting proteins is needed to understand the functional differences and 
targeting mechanisms of H2A and H2A.Z. However, current AP-MS strategies suffer from several limitations: (1) weak interactions may not be detected; (2) interactions that require a physiological chromatin environment may not be detected; (3) some proteins detected as interacting with H2A.Z may not interact directly but instead their interaction may be mediated by other components of the H2A.Z-containing nucleosomes; (4) some detected interactions may occur only in vitro but not in vivo; and (5) the proteins pulled down by an antibody vary depending on the binding and washing conditions, which may result in very low reproducibility of data from different experiments in the same lab or between labs even if the same antibody is being used.

Because a comprehensive catalog of H2A- and H2A. $\mathrm{Z}$-interacting proteins is needed to understand their functional differences, we developed a sensitive in vivo strategy, termed bPPI-seq (bait Protein-Protein Interaction-sequencing), to detect genome-wide interacting proteins and used this strategy to compare the H2A- and H2A.Z-interacting proteins genome-wide. Our results indicated that H2A.Z-interacting proteins are enriched for proteins that regulate transcription while such proteins do not constitute the $\mathrm{H} 2 \mathrm{~A}$-interacting proteins, thus providing novel insights into the mechanisms underlying their functional difference. We found that H2A.Z interacts with 20 DNA-binding transcription factors and further demonstrate that Osr1, a zinc-finger transcription factor, facilitates targeting of H2A.Z to regions containing Osr1 motifs, providing evidence that sequence-specific transcription factors mediate targeting of H2A.Z to specific chromatin regions. Our results indicate that bPPI-seq is an effective strategy to identify genome-wide protein interactions in mammalian cells.

\section{Results}

Reconstituting active GFP by fusion of its $N$-terminus with H2A.Z and its C-terminus with ANP32E

An active GFP can be reconstituted from two non-fluorescent fragments of GFP brought together by two interacting proteins [19]. Using this strategy, $\alpha$-actinin 4 (ACTN4) was identified as a substrate of the serine/threonine protein kinase AKT1, which mediates its translocation to cell membrane [20]. To test if an active GFP can be reconstituted from two fragments fused to H2A.Z and an H2A.Z-interacting protein, we co-expressed the fusion protein (GFPN-H2A.Z) between GFP N-terminus and H2A.Z, and the fusion protein (ANP32E-GFPC) between GFP C-terminus and ANP32E, a known H2A.Z-interacting protein $[16,17]$ in NIH 3 T3 cells. The cells turned green after $48 \mathrm{~h}$ of expression (Figure 1A, left panel), while very few green cells were detected when GFPN-H2A.Z was co-expressed with a non-interacting protein Tinf2-GFPC (Figure 1A, right panel). These results indicate that H2Aand H2A.Z-interacting proteins can be detected by the GFP reconstitution assay in vivo.

\section{Random gene tagging with ERM vectors}

To unbiasedly identify H2A- and H2A.Z-interacting proteins, we randomly tagged endogenous genes by exon trapping using an enhanced retroviral mutagen vector [21], which contains the cDNA of GFPC and a splicing donor (SD) driven by a Doxycycline-inducible promoter. After integration upstream or inside an endogenous gene, the splice donor mediates the formation of a hybrid transcript of GFPC fused in frame to the downstream endogenous exons, thus expressing a GFPC fusion protein.

\section{Ectopically expressed GFPN-H2A.Z behaves similarly to endogenous $H 2 A . Z$}

To identify all proteins that interact with $\mathrm{H} 2 \mathrm{~A}$ and H2A.Z, first, we established three stable cell lines of GFPN, GFPN-H2A and GFPN-H2A.Z in which the expression levels of GFPN-H2A or GFPN-H2A.Z were comparable to that of endogenous $\mathrm{H} 2 \mathrm{~A}$ or $\mathrm{H} 2 \mathrm{~A} . \mathrm{Z}$ (Supplementary information, Figure S1). Furthermore, GFPN-H2A.Z in the GFPN-H2A.Z stable cell line was incorporated into chromatin and enriched in transcriptional regulatory regions in a similar way to endogenous H2A.Z, as shown by the genome browser snapshots, TSS profile analysis and Venn diagrams (Figure 2).

To further test whether GFPN-H2A.Z shares similar functions with endogenous H2A.Z, we chose to perform rescue experiments by ectopically expressing GFPN-H2A.Z in H2A.Z knockdown cells. First, we compared the chromatin binding patterns of ectopic GFPN-H2A.Z with those of endogenous H2A.Z using ChIP-seq by titrating the concentration of transfected GFPN-H2A.Z construct. From a wide range of DNA amounts used for transfection $(0,0.75,1.5,3.0,6.0,12$ $\mu \mathrm{g}$ ), we found that although there is some increased background binding, the GFPN-H2A.Z peaks still correspond closely to endogenous H2A.Z peaks, as shown by the genome browser snap shots, TSS profile analysis and Venn diagrams (Supplementary information, Figures S2 and S3). Thus, we chose to use $3.0 \mu \mathrm{g}$ of the GF$\mathrm{PN}-\mathrm{H} 2 \mathrm{~A} . \mathrm{Z}$ construct in the rescue experiment. While the ectopic expression of GFPN-H2A.Z somehow suppressed the expression of endogenous H2A.Z in the cells, the total H2A.Z level (GFPN-H2A.Z + H2A.Z) in transfected cells was about two-fold of the H2A.Z level in control cells by western blotting (Supplementary information, Figure S4A). Finally, GFPN-H2A.Z rescued the knockdown of 

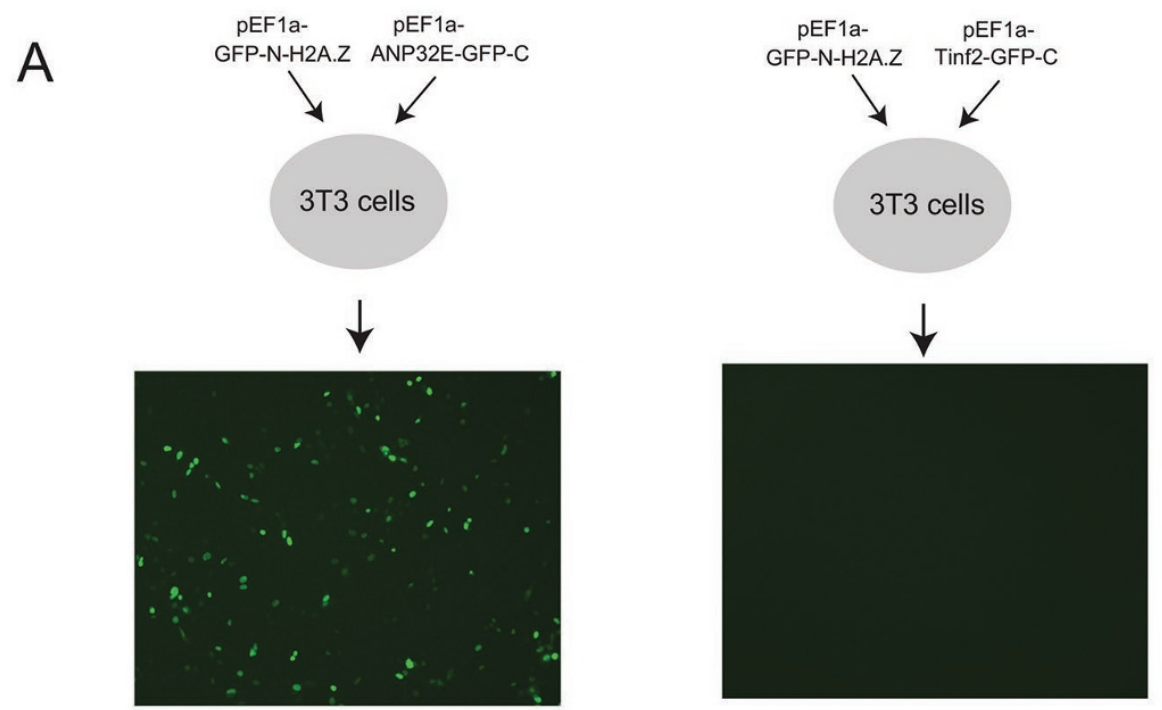

B
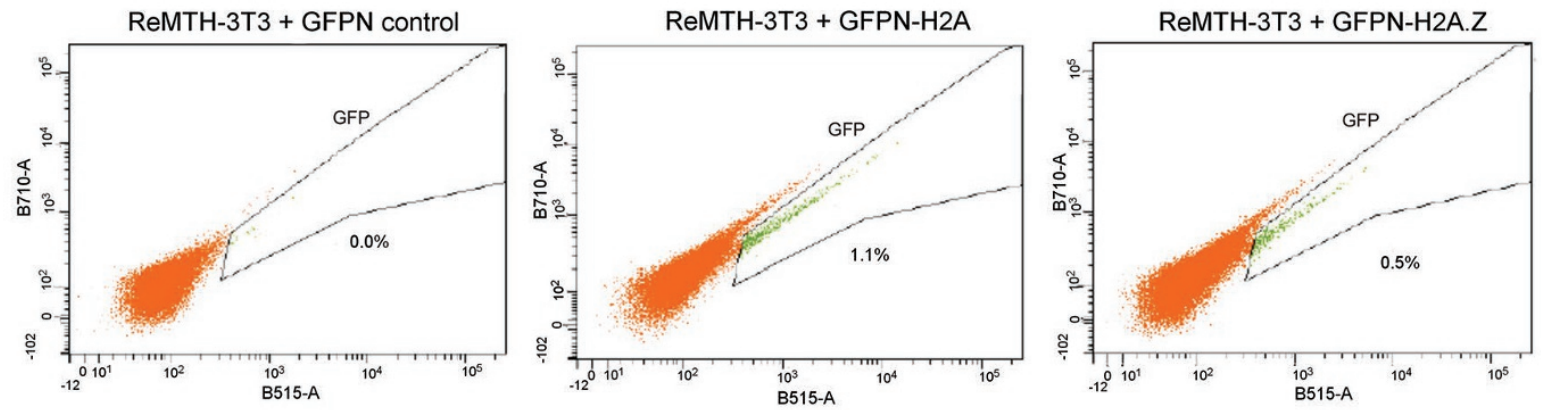

C

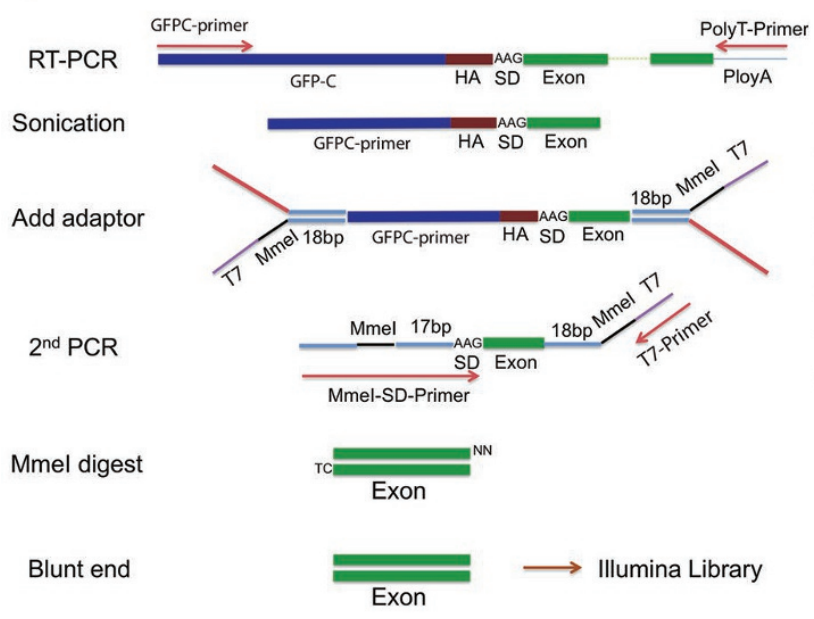

D

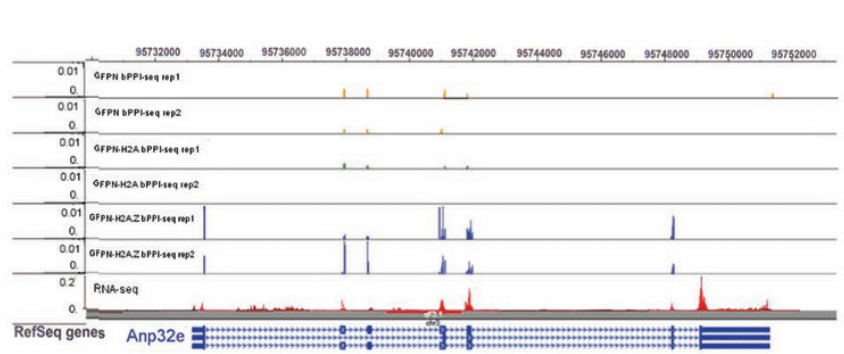

endogenous $H 2 A . Z$ assessed using reverse-transcription qPCR assays (Supplementary information, Figure S4B). These results indicate that GFPN-H2A.Z functions similarly with the endogenous H2A.Z.

Genome-wide identification of H2A- and H2A.Z-interacting

proteins

To identify H2A- and H2A.Z-interacting proteins, the stable cell lines expressing GFPN alone, GFPN-H2A and GFPN-H2A.Z were infected with virus carrying three reading frames of GFPC (ReMTH-GFPC1, 2 and 3 viral particles) to tag endogenous genes with the GFPC 
Figure 1 Genome-wide detection of H2A- and H2A.Z-interacting proteins using bPPI-seq. (A) Co-expression of GFPN-H2A.Z with ANP32E-GFPC reconstitutes an active green fluorescent protein. The constructs expressing full-length fusion proteins in pEF1a vector were co-transfected into NIH 3T3 cells and examined under microscope $48 \mathrm{~h}$ post transfection. Tinf2-GFPC is used as a negative control. (B) Isolation of GFP+ cells by flow cytometry sorting. The stable cell lines expressing GFPN-H2A.Z, GFPN-H2A or GFPN alone were transduced with three ReMTH-GFPC1, 2 and 3 viral particles to tag endogenous genes with GFPC domain, selected by puromycin for 5 days, followed by Doxycycline induction for $48 \mathrm{~h}$ before sorting. (C) Flow chart for preparation of the H2A.Z-interacting protein libraries (bPPI-seq) from RNAs isolated from the sorted cells. (D) Genome Browser image showing the bPPI-seq reads in the gene encoding H2A.Z-interacting protein ANP32E. The $y$-axis was normalized to library sequencing depth. The peak location indicates the fusion site of hybrid mRNA between C-terminal half (GFPC) and the mRNA of endogenous gene. The panels from top to the bottom are bPPI-seq GFPN (two replicates), bPPI-seq GFPN-H2A (two replicates), bPPI-seq GFPN-H2A.Z (two replicates) and RNA-seq.

domain. Following puromycin selection and subsequent induction by Doxycycline treatment for $48 \mathrm{~h}$, green cells were collected by flow cytometry sorting. About $0.5 \%$ or $1.1 \%$ of cells expressing GFPN-H2A.Z or GFPN-H2A were GFP-positive, while very few cells expressing the GFP N-terminal domain showed green signals (Figure 1B). To identify the genes encoding H2A- or H2A.Z-interacting proteins, total RNAs from 50 to $150 \mathrm{~K}$ sorted cells were reverse transcribed using oligo dT primers and fragmented to $200-500$ bp by sonication (Figure 1C). Following end repair and ligation of a Y-shaped T7 adaptor containing a $\mathrm{MmeI}$ recognition site to both ends, the DNA fragments between 250 and 650 bp were isolated from gels. In the second PCR using a T7 primer and three Mme-SD-primers for the three reading frames of the GFPC fusion vector, fusion cDNAs containing HA and SD sequences were amplified. Digestion with MmeI cleaves DNA 20 bp away from its recognition site and thus was used to remove the HA and T7 primer sequences. The resulting DNA fragments were blunt-ended and used to construct RNA-seq libraries (Figure 1C). Six bPPI-seq libraries were pooled and sequenced on Hiseq2500. The short reads were mapped onto the mouse genome sequence to identify the genes encoding the interacting proteins. As expected, ANP32E was detected as a protein that specifically interacts with H2A.Z (Figure 1D). The location of short sequence reads reveals the fusion sites of the endogenous mRNA with GFPC, which are indicated by sharp signals at the exons where fusion occurs, as shown in the genome browser images (Figure 1D). Fusion events may occur at different exons of a gene as indicated by multiple peaks along the gene (Figure 1D). The fusion sites provide information on the location of a protein domain that is required for interacting with the bait protein in the cells. Because of the requirement of spatial proximity for reconstitution of an active GFP from the two GFP fragments, most proteins detected by this strategy show direct interactions with the bait.

To identify H2A- and H2A.Z-interacting proteins, we aligned all reads from the three bPPI-seq libraries, in- cluding GFPN, GFPN-H2A and GFPN-H2A.Z, to mouse genome mm9 using TopHat [22]. The read density in the coding regions of genes was computed for each library. Genes were required to satisfy several criteria to be classified as potential interacting proteins (see Materials and Methods). A significant feature allowing a protein to be considered as interacting with $\mathrm{H} 2 \mathrm{~A}$ or H2A.Z was if the difference of read density of the gene was significant in comparison to the GFPN control library, computed using the method of Audic and Claverie [23] (false-positive discover rate $<0.000001$; fold-change of read densities over eight-fold.)

Using the above criteria, we identified 217 and 295 H2A.Z- and H2A-interacting proteins, respectively (Figure 3A; Supplementary information, Tables S1, S2). Overall, the average molecular weights of H2A.Z- and H2A-interacting proteins were comparable to those of all proteins (Supplementary information, Figure S5; Supplementary information, Tables S3, S4). Among the H2A.Z-interacting proteins identified by our bPPI-seq assay, we found six proteins, including Anp32e, Actb, H2afz, Phf14, Mbtd1 and Smarca4, that had been discovered by mass spectrometry analysis [14, 16, 24] and co-IP [11] as H2A.Z partners, indicating that the assay is able to detect previously identified interacting proteins.

To test the reproducibility of bPPI-seq libraries, we plotted the logarithm of read density between two biological replicates (Figure 3B). The replicate samples are highly correlated ( $r=0.78,0.83$, respectively).

\section{H2A.Z-interacting proteins}

Among the 217 H2A.Z-interacting proteins, 131 (60\%) are nuclear proteins and the rest are cytoplasmic proteins. This is biologically relevant since H2A.Z functions in the nucleus. It is likely that the cytoplasmic proteins interact with H2A.Z during its translation in the cytoplasmic compartment and/or during its transportation from cytoplasm to nucleus. It is also possible that some cytoplasmic proteins may have unknown functions in the nucleus where they interact with H2A.Z. 
A

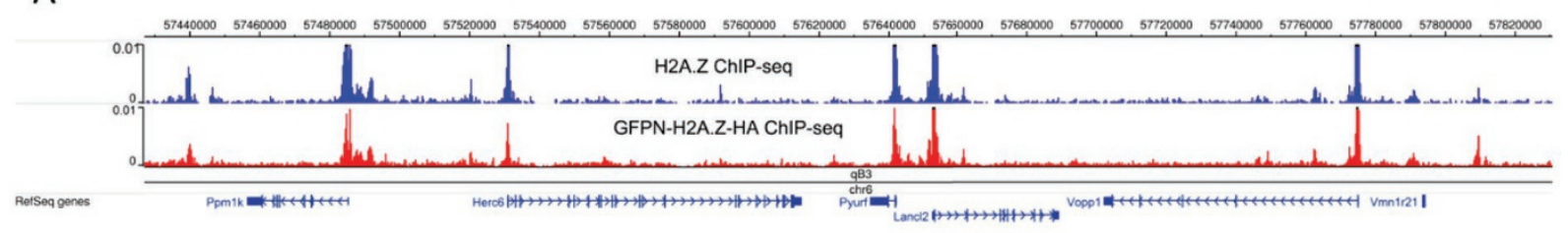

B
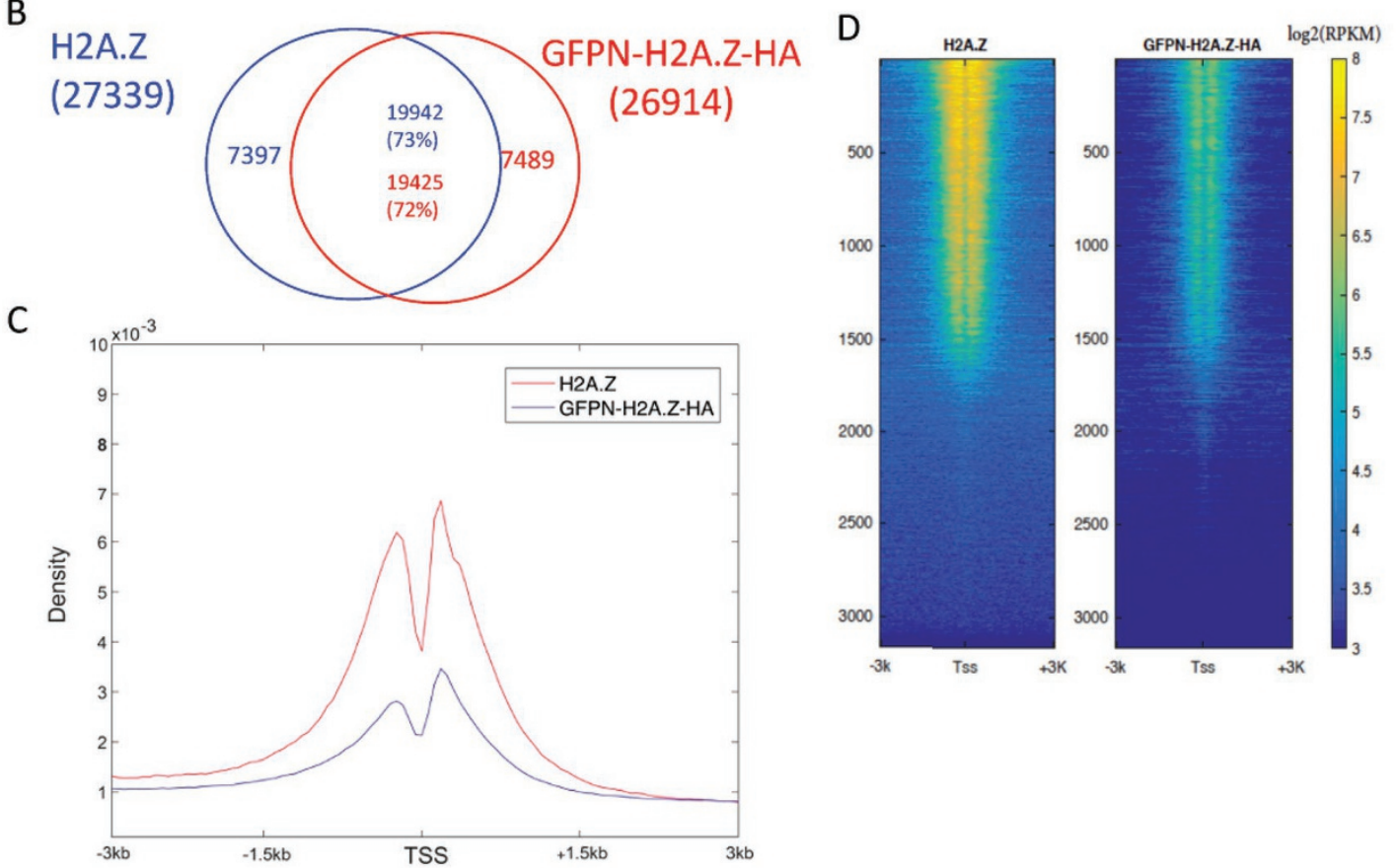

Figure 2 GFPN-H2A.Z is incorporated into chromatin with enrichment in transcriptional regulatory regions similar to endogenous H2A.Z. (A) A snapshot of genome browser image of ChIP-seq data for endogenous H2A.Z and GFPN-H2A.Z-HA. The top panel corresponds to H2A.Z ChIP-seq in shLuc control cells. The bottom panel corresponds to HA CHIP-seq in the GFPN-H2A.Z-HA stable cell line. (B) Venn diagram showing that H2A.Z- and GFPN-H2A.Z-HA-enriched regions highly overlap. (C) Average TSS profile plots for the tag density of the H2A.Z ChIP-seq in control cells and HA ChIP-seq in the GFPN-H2A.Z-HA stable cell line. (D) TSS profile plots for the logarithm of the density of the H2A.Z and GFPN-H2A.Z-HA ChIP-seq experiments.

The bPPI-seq data indicated an interaction between H2A.Z and Smarca4 in accord with previous co-IP data [11] showing H2A.Z interacts with Smarca4 in mouse endoderm/hepatic progenitor cells, where H2A.Z and Foxa2 recruit nucleosome remodeling complexes including Smarca4, enabling nucleosome depletion and ES cell differentiation [11]. We also detected an interaction of H2A.Z with INO80, previously shown to mediate the deposition of H2A.Z into chromatin in both yeast and human cells $[18,25-30]$. The identification of the interaction between INO80 and H2A.Z in our mouse data would suggest the conservation of these interactions and that the promotion of homologous recombination in mouse by INO80 via the removal of H2A.Z occurs similarly to the analogous process in humans.

In addition to the above examples, we also found three data sets in the literature reporting identification of proteins co-purifying with the H2A.Z nucleosomes using affinity purification followed by mass spectrometry analysis $[14,16,24]$. These studies identified 45,55 and 75 , proteins, respectively with only seven identified proteins being shared among the three studies (Supplementary information, Table S5) that are mainly components of the SRCAP and TIP60 complexes [14, 16, 24]. ANP32E is a component of these complexes, which directly interacts with H2A.Z and functions as an H2A.Z chaperone [16]. The low reproducibility of these different data sets indicates the high variability of the strategy, which may re- 

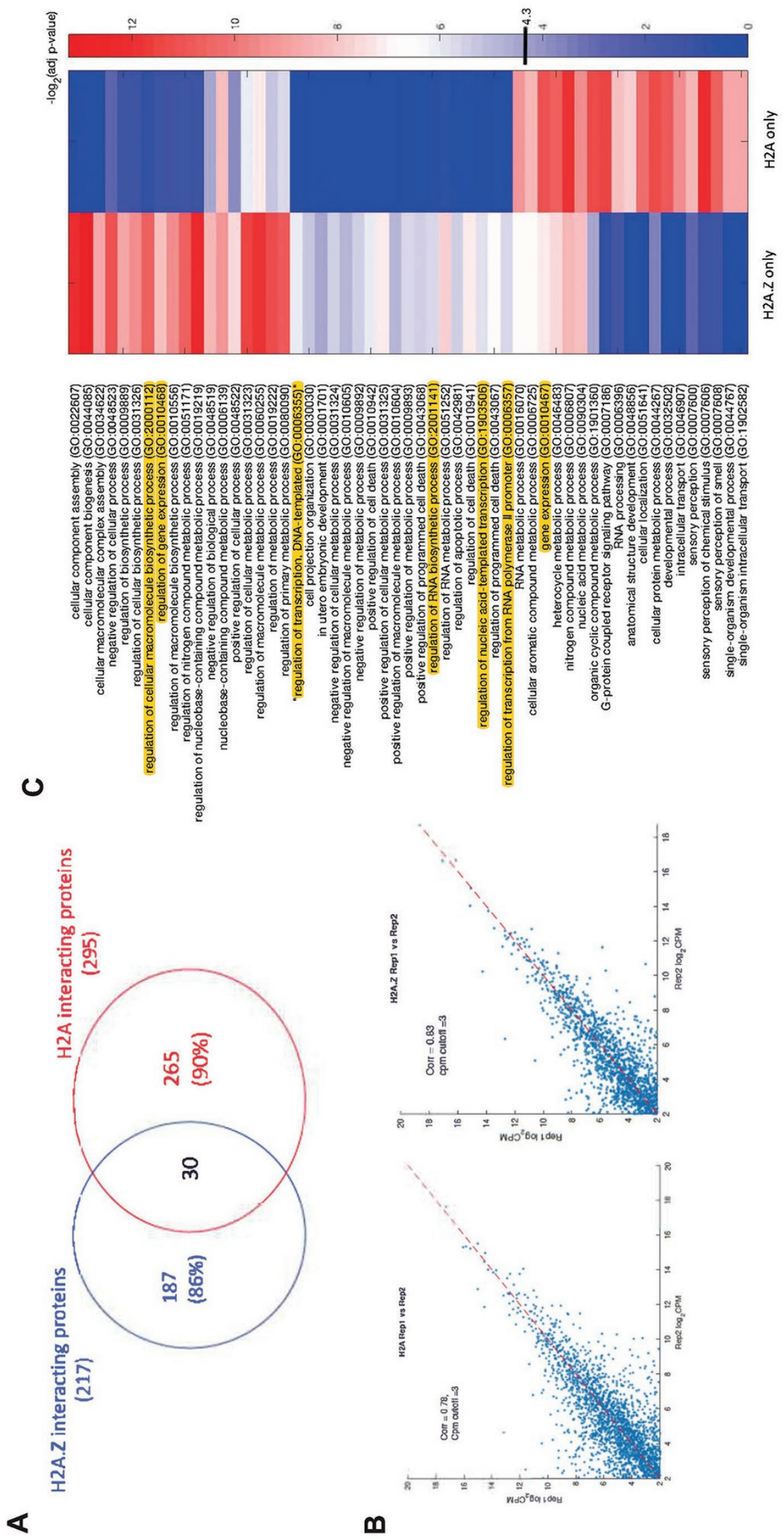

www.cell-research.com | Cell Research | SPRINGER NATURE 
flect experimental conditions. Among the proteins identified by AP-MS include the H2A.Z-chaperone ANP32E, of which the direct interaction with H2A.Z is unarguably supported by crystallographic data [16, 17]. Consistently, our bPPI-seq data also revealed these proteins as H2A. Z-interacting proteins (Supplementary information, Table S5). In addition, bPPI-seq also identified H2A.Z itself as an H2A.Z-interacting protein. This may be an indication of the direct contact of the two H2A.Z peptides within a nucleosome core particle as revealed by its crystal structure [13]. Together these results indicate that proteins known to directly interact with H2A.Z can be identified by both AP-MS and bPPI-seq.

\section{$H 2 A$ and $H 2 A . Z$ interact with distinctive protein sets}

$\mathrm{H} 2 \mathrm{~A}$ is a structural protein for nucleosome assembly while H2A.Z is involved in setting up chromatin structure at regulatory regions required for transcriptional activation or repression [8]. Identification of their interacting partners in the nucleus may shed light on the mechanisms underlying functional differences between them. Among the H2A- and H2A.Z-interacting proteins, there are $265 \mathrm{H} 2 \mathrm{~A}$-specific and $187 \mathrm{H} 2 \mathrm{~A}$.Z-specific proteins, respectively. To gain a perspective of the biological processes in which they might function, we performed a gene ontology analysis [31] upon both sets of interacting proteins that we identified. This revealed the H2A.Z-interacting proteins and $\mathrm{H} 2 \mathrm{~A}$-interacting proteins to be involved in distinct biological processes (Figure 3C). Gene ontology terms were manually selected reflecting transcriptional regulation based on their definitions and are highlighted in Figure 3C. The H2A.Z-interacting proteins are enriched in those with transcriptional-related terms in contrast to $\mathrm{H} 2 \mathrm{~A}$-interacting proteins (Figure 3C). Among the H2A.Z-interacting proteins are 20 transcription factors including Osr1, a protein that may facilitate the targeting of H2A.Z or enable the downstream events of transcriptional activation by recognizing specific DNA sequences. These results are consistent with the previous observation that H2A.Z nucleosomes are involved in the regulation of transcription.

\section{Validation of H2A.Z-interacting proteins}

To verify that the identified proteins do interact with $\mathrm{H} 2 \mathrm{~A} . \mathrm{Z}$, we randomly selected 24 proteins from the H2A.Z-interacting protein list and generated the GFPC fusion protein expression constructs, including the zinc-finger transcription factor Osr1. We also selected an H2A.Z-interacting protein ANP32E, and a non-interacting protein Tinf 2 as the respective positive and negative controls. As expected, transfection of the ANP32E construct into the GFPN-H2A.Z stable cell line gave rise to green fluorescence (Figure 4A) whereas the negative control Tinf 2 did not. Among the 24 randomly selected proteins, 19 of them $(79 \%)$ gave rise to green fluorescence (Figure 4A; Supplementary information, Table S6). To further validate the interactors using a different strategy, we performed co-immunoprecipitation assays. We found all the 14 GFPC fusion proteins that we tested could co-immunoprecipitate with GFPN-H2A.Z (Figures 4B and 5A). Notably, although four of the tested proteins have been defined as cytoplasmic proteins in the literature, they displayed nuclear GFP signals in our assay (Figure 4A; Supplementary information, Table S6) and also co-immunoprecipitated with GFPN-H2A.Z (Figure $4 \mathrm{~B})$. Therefore, these results validate the proteins identified by bPPI-seq as H2A.Z-interacting proteins and so indicate that bPPI-seq is a robust technique for detecting bait protein-protein interactions in vivo.

\section{Osrl directly interacts with $H 2 A . Z$ in vivo and in vitro}

The SWR1 and INO80 complexes are responsible for deposition of H2A.Z into chromatin in yeast [25-30]. In mammalian cells, the SRCAP, p400/TIP60 complexes, INO80 chromatin remodeler, and the H2A.Z-specific chaperone, ANP32E $[14,16,17,24]$ are implicated in mediating H2A.Z's deposition into chromatin. However, these proteins do not have sequence-specific DNA-binding activities and thus it remains unclear how H2A.Z finds its remarkably specific target sites on chromatin. H2A.Z was reported to act together with the Foxa2 transcription factor to regulate nucleosome structure and gene activation [11], suggesting that sequence-specific

Figure 3 H2A- and H2A.Z-interacting proteins are enriched in distinctive biological processes. (A) Venn diagram showing that $\mathrm{H} 2 \mathrm{~A}$ and $\mathrm{H} 2 \mathrm{~A}$.Z interact with different sets of proteins. (B) Detection of $\mathrm{H} 2 \mathrm{~A}$ - and $\mathrm{H} 2 \mathrm{~A}$.Z-interacting proteins by bPPI-seq is highly reproducible between replicate experiments. The $\log _{2} \mathrm{rpkm}$ values of mRNAs of interacting proteins between replicate experiments are compared in scatter plots. (C) A heat map showing the significance of the gene ontology (GO) terms for the $\mathrm{H} 2 \mathrm{~A}$.Z-interacting proteins and $\mathrm{H} 2 \mathrm{~A}$-interacting proteins. $\mathrm{H} 2 \mathrm{~A}$-interacting proteins and $\mathrm{H} 2 \mathrm{~A}$.Z-interacting proteins are involved in distinct biological processes. Highlighted terms are transcription-related terms based on the definitions of $\mathrm{GO}$ terms. The color level represents the negative logarithm of the adjusted $P$-value for the significance of the GO terms. The intensity of red represents greater significance of the $\mathrm{GO}$ terms whereas blue represents less significant $\mathrm{GO}$ terms. A cutoff of color level $=4.3$ (corresponding to a $P$-value of 0.05 ) is applied to consider if the GO terms are statistically significant for the gene sets. 

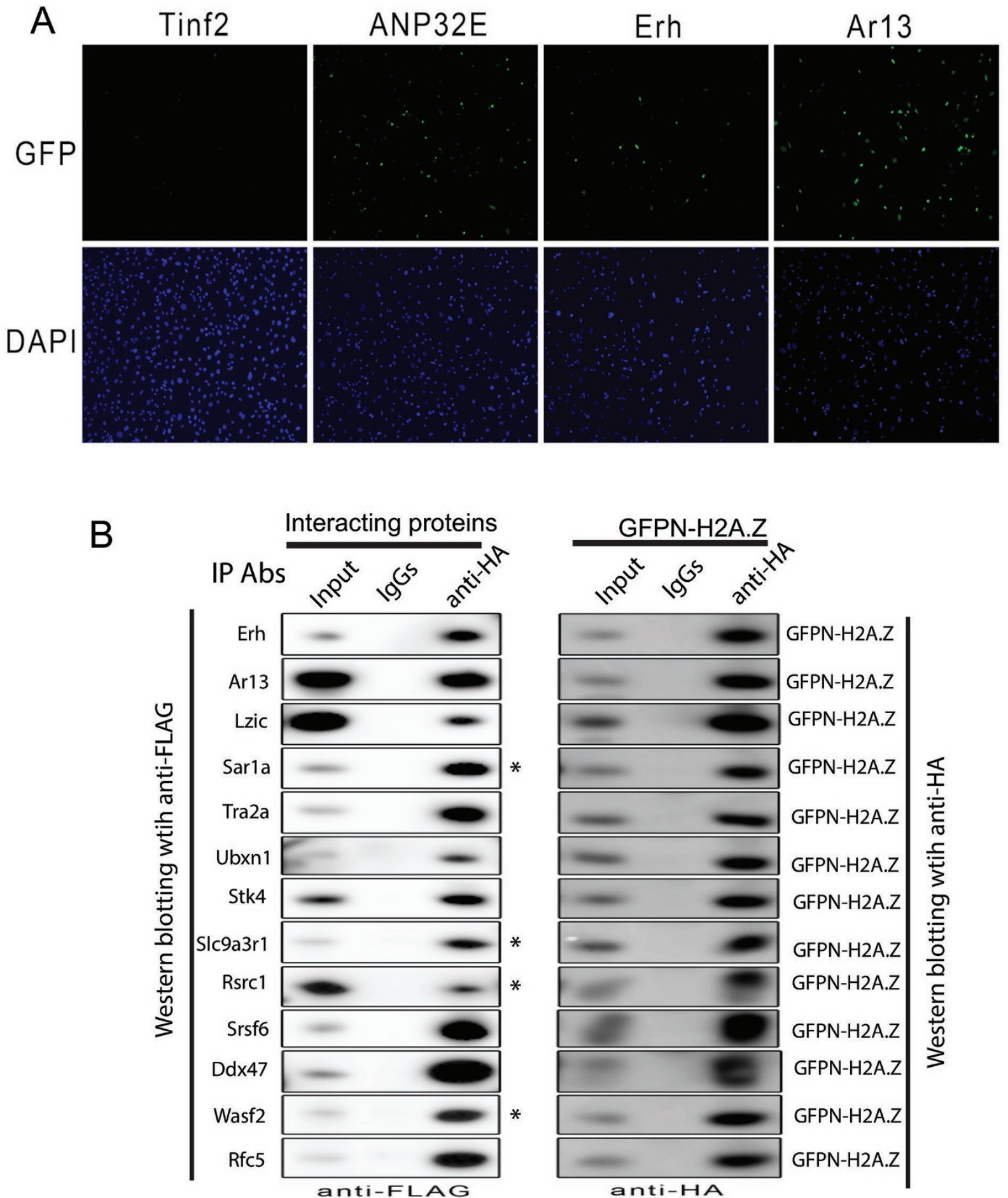

Figure 4 Validation of the H2A.Z-interacting proteins. (A) Reconstitution of active GFPs by co-expression of the fusion proteins GFPC-H2A.Z interactor and GFPN-HA-H2A.Z. The construct expressing the fusion protein between GFPC and an H2A.Z-interacting protein was transfected into the stable cell line expressing GFPN-HA-H2A.Z fusion protein and induced using Doxycyline for $48 \mathrm{~h}$. Images of GFP and DAPI staining signals were taken (10x). (B) Co-IP of GFPN-HA-H2A.Z with the fusion protein between GFPC and H2A.Z-interacting proteins. Whole-cell extracts were made from the cells expressing the GFPN-HA-H2A.Z and its interacting fusion proteins as described in (A) and immunoprecipitated using HA antibody. The immunoprecipitates were resolved by SDS-PAGE and blotted by anti-FLAG antibody that detects the H2A.Z interactor fusion protein (left panel) and HA antibody that detects the GFPN-HA-H2A.Z fusion protein (right panel), respectively. The asterisks indicate cytoplasmic proteins based on literature. 
DNA-binding factors may contribute to its targeting on chromatin. To test whether sequence-specific factors facilitate the binding of H2A.Z to its specific target sites, we further validated the interaction between H2A.Z and Osr1. NIH 3 T3 cells were transfected with GFPCFLAG-Osr1 and either GFPN-HA-H2A or GFPN-HAH2A.Z for 2 days and cell extracts from these cells were subjected to immunoprecipitation with either anti-FLAG or anti-HA antibodies. As expected, both GFPN-HAH2A and GFPN-HA-H2A.Z nucleosomes were efficiently pulled down by anti-HA antibody (Figure 5A, top panel, lanes 2, 4, 6, 8). However, only H2A.Z but not H2A was co-immunoprecipitated with Osr1 by anti-FLAG antibody (Figure 5A, top panel, compare lanes 1 and 3 with 5 and 7). This is consistent with the observation that Osr1 interacts specifically with H2A.Z as we had detected by the bPPI-seq assay. As a negative control, we found that Tinf2-FLAG-GFPC was not co-immunoprecipitated with either H2A or H2A.Z (Figure 5A, bottom panel). These results further confirmed that Osr1 specifically interacts with H2A.Z in the cells. To further test whether H2A.Z directly interacts with Osr1 and the domains involved in the interaction, we expressed and purified the full-length and different domains of H2A.Z and Osr1 in bacteria (Figure $5 \mathrm{~B}$ ) and examined their interactions in vitro (Figure $5 \mathrm{C}$ and $5 \mathrm{D}$ ). We immobilized either GST or GST-Osr1 fusion protein on glutathioine beads and found that the latter was able to bind H2A.Z and that this required its middle domain (amino acids 41-80) (Figure 5C). However, in order to recapitulate full binding, both the middle and $\mathrm{C}$-terminal domains of H2A.Z were needed, consistent with previous findings that the acid patch M6 region located in the $\mathrm{C}$-terminal part is crucial for the function of H2A.Z and its interaction with other proteins $[3,16,29,32,33]$. By comparison, both the $\mathrm{N}$ - and $\mathrm{C}$-terminal domains of Osr1 exhibited binding to MBPH2A.Z beads (Figure 5D).

\section{Osr1 and H2A.Z co-localize on chromatin and regulate} shared targets

The finding that Osr1 and H2A.Z interact directly both in vitro and in vivo suggests that they may co-localize on chromatin. Indeed, ChIP-seq assays indicated that H2A.Z and Osr1 co-localize extensively on chromatin (Figure 6A and 6B). We found that $60 \%$ of the co-localizing regions for H2A.Z- and Osr1 corresponded to promoters, whereas $80 \%$ of regions occupied only by H2A.Z corresponded to enhancers (Supplementary information, Figure S6A). Interestingly, the promoters bound by both H2A.Z and Osr1 exhibited significantly higher expression levels than the promoters bound by H2A.Z alone (Supplementary information, Figure S6B).
These results suggest that H2A.Z and Osr1 may regulate the expression of shared target genes. To test this hypothesis, we knocked down either $H 2 A . Z$ or $O s r l$ and analyzed the mRNA expression profiles in the knockdown cells using RNA-seq. Knocking down H2A.Z led to the upregulation of 1799 genes and downregulation of 2346 genes (Supplementary information, Table S7). Knocking down Osrl led to 927 genes being up-regulated and 1051 down-regulated genes (Supplementary information, Table S8). Interestingly there was a significant correlation between the up- and down-regulation of genes in response to knockdown of $H 2 A . Z$ or $O s r l$ (Figure 6C) and large degrees of overlap (Figure 6D and $6 \mathrm{E})$, suggesting that they not only co-localize to the same target regions on chromatin but also contribute to the expression of the same set of target genes.

\section{Osr 1 contributes to H2A.Z targeting on chromatin}

To test whether Osr1 contributes to the deposition of H2A.Z on chromatin, we analyzed the ChIP-Seq binding profiles of H2A.Z in control and Osrl knockdown cells. Knockdown of $\mathrm{Osrl}$ resulted in 112 significantly decreased and 26 significantly increased H2A.Z binding peaks, respectively (Supplementary information, Table S9). Using the genomic SELEX method [34] with bacterially expressed Osr1 protein, we identified a consensus binding motif for Ors1 (Figure 7A), which is similar to previously reported Osr1 motif [35]. We found that 40\% of the increased peaks of H2A.Z association had the Osr1 motif whereas a significantly larger fraction $(69 \%)$ of the decreased H2A.Z peaks contained the Osr1 motif (Figure 7B and 7C). This suggests that the presence of an Osr1 motif may direct Osr1 binding to the site and influence the targeting of H2A.Z. To confirm that Osr1 binding is indeed related to the observed decrease in H2A.Z targeting in the Osrl knockdown cells, we separated the H2A.Z peaks into two groups: one with Osr1 binding and the other without Osr1 binding based on Osr1 ChIPseq data (Figure 7D). Interestingly, the same trend was observed as using the Osr1 motif (Figure 7E), indicating that the sequence-specific recognition of DNA by Osr1 contributes to the targeting of H2A.Z to chromatin.

\section{Discussion}

The canonical core histone $\mathrm{H} 2 \mathrm{~A}$ is a structural subunit for nucleosome assembly and packaging of DNA in the nucleus. Displacement of H2A with the variant H2A.Z is associated with nucleosome destabilization and chromatin accessibility at regulatory regions of transcription such as enhancers and promoters. While these two closely related proteins differ by $30 \%$ in amino-acid sequence, 


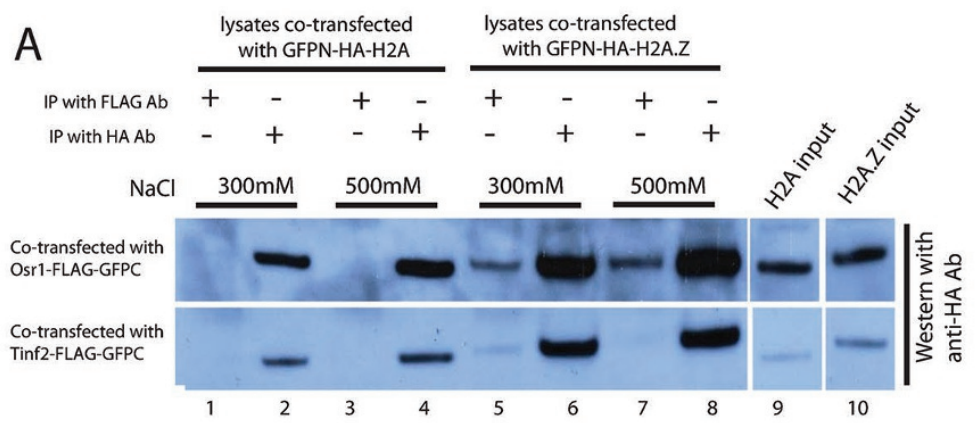

B

MBP-H2A.Z fusion proteins

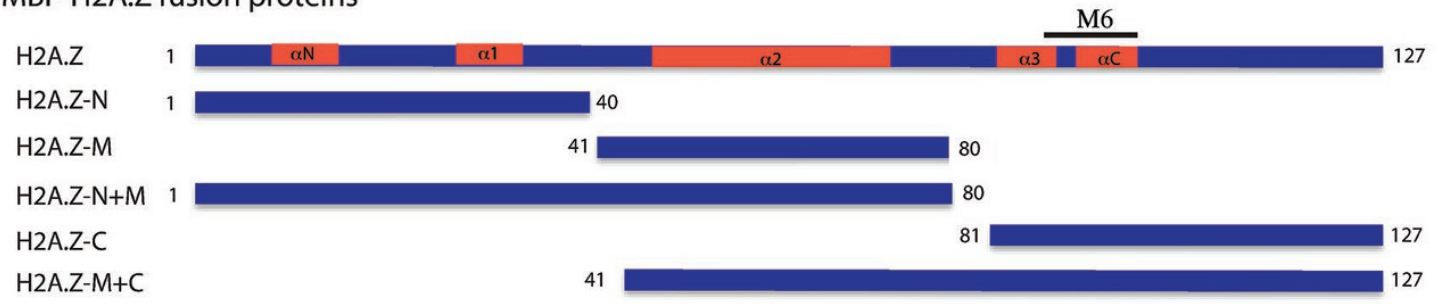

GST-Osr1 fusion proteins
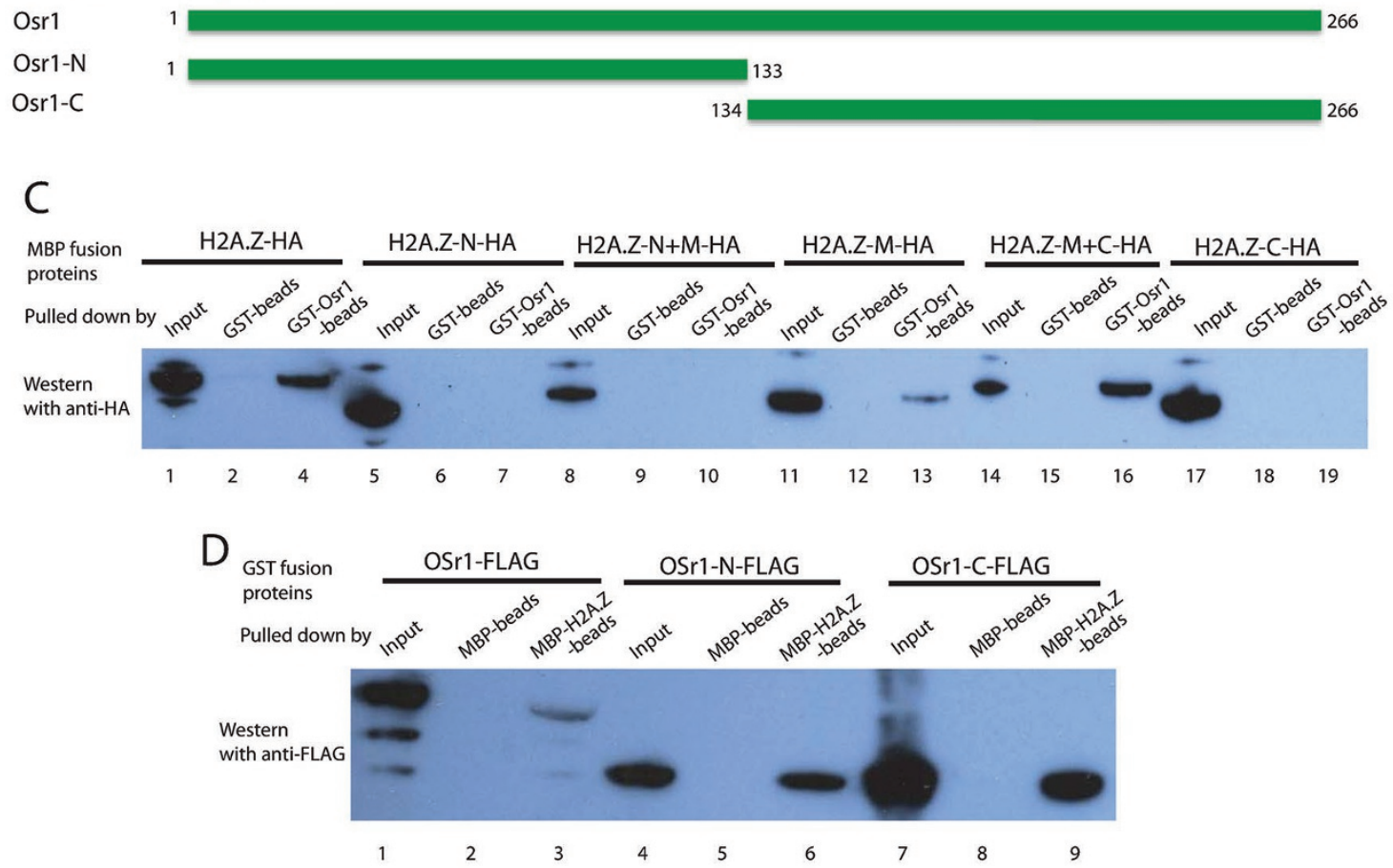

Figure 5 Osr1 interacts directly with H2A.Z in vivo and in vitro. (A) Osr1 co-immunoprecipitates with H2A.Z but not H2A from cell extracts. Whole-cell extracts were prepared from NIH3T3 cells co-transfected with Osr1-FLAG-GFPC or Tinf2-FLAG-GFPC and GFPN-HA-H2A or GFPN-HA-H2A.Z for $48 \mathrm{~h}$, followed by immunoprecipitation using anti-FLAG or anti-HA antibodies. The immunoprecipitates were washed with buffers containing 300 or $500 \mathrm{mM} \mathrm{NaCl}$ and detected by western blotting with anti-HA antibody. (B) Cartoons showing the full length and truncated H2A.Z proteins expressed in bacteria as MBP fusion proteins, which were purified using Amylose beads. The helices and M6 acid patch domains are indicated. The full length and different truncated Osr1 proteins were expressed in bacteria as GST fusion proteins and purified using glutathione-Sepharose 4B beads. (C) GST or GST-Osr1 fusion proteins bound to beads were incubated with different MBP-H2A.Z fusion proteins. The H2A.Z proteins captured by the beads were detected by western blotting using anti-HA antibody. (D) MBP or MBPH2A.Z fusion proteins bound to beads were incubated with different GST-Osr1 fusion proteins. The Osr1 proteins captured by the beads were detected by western blotting using anti-FLAG antibody. 


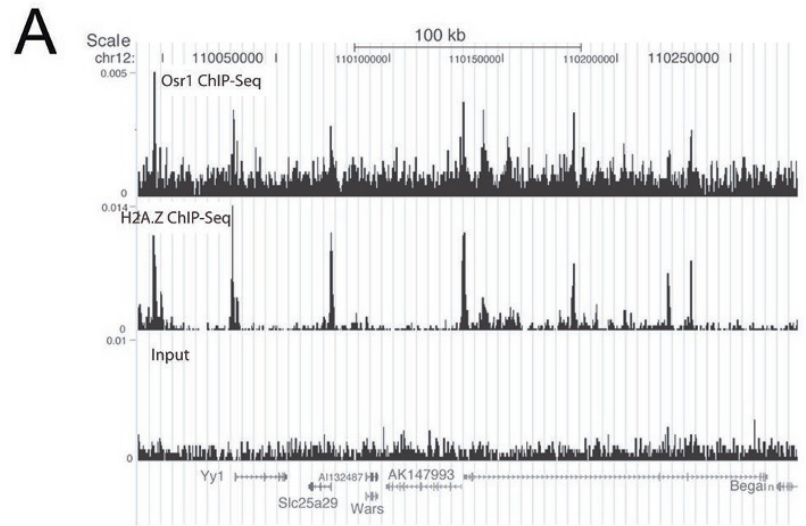

C

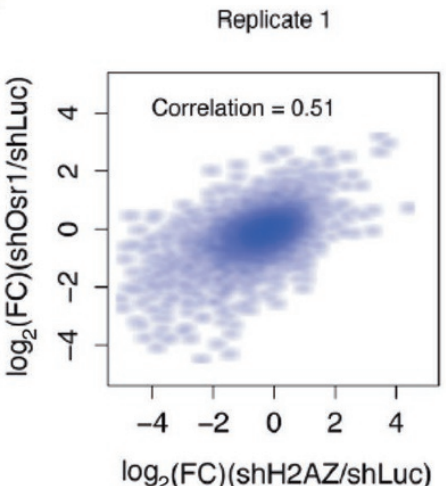

B

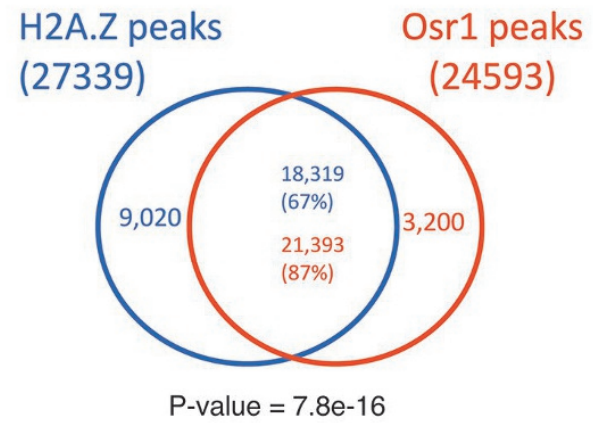

$\mathrm{E}$

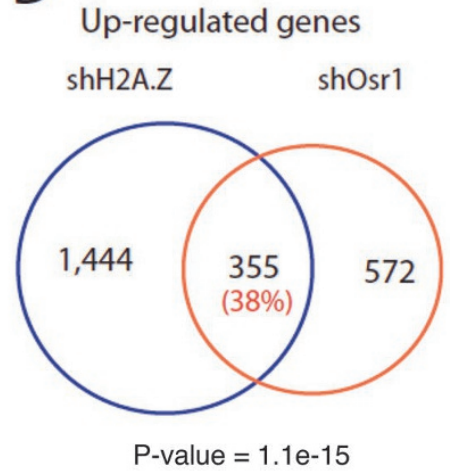

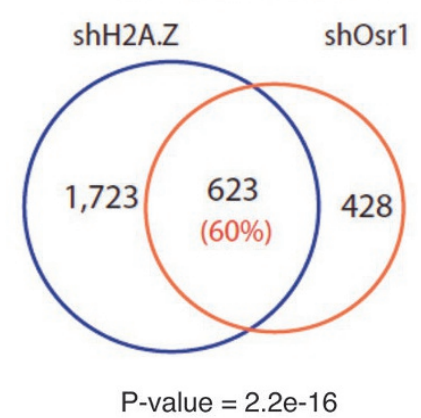

Figure 6 Osr1 facilitates H2A.Z targeting and co-regulates gene expression with H2A.Z. (A) Genome browser image showing the co-localization of H2A.Z and Osr1 ChIP-Seq signals on chromatin. (B) Venn diagram showing the overlap of Osr1 and $\mathrm{H} 2 \mathrm{~A} . \mathrm{Z}$ peaks in the genome. The number in red (blue) in the intersection region refers to the number of Osr1 (H2A.Z) peaks overlapping with H2A.Z (Osr1) peaks. The difference between the number of overlapping peaks is due to the overlap between multiple Osr1 peaks and a single H2A.Z peak. The $P$-value for the significance of overlap was calculated by using the hypergeometric distribution, in which the smaller number of overlapped peaks was used. (C) The changes in gene expression by knocking down H2A.Z and Osr1 are highly correlated. The gene expression in shLuc, shH2A.Z and shOsr1 cells was measured using RNA-seq. The fold change of gene expression (FC) was calculated for shH2A.Z and shOsr1 by comparing to shLuc. (D) Venn diagram showing the overlap of up-regulated genes after knocking down $H 2 A$.Z and Osr1. The $P$-values were calculated by using the hypergeometric distribution with 22490 total genes. (E) Venn diagram showing the overlap of down-regulated genes after knocking down H2A.Z and Osr1. The P-value was calculated by using the hypergeometric distribution with 22490 total genes.

what underlies their functional difference is not fully clear. In this study, we developed the novel bPPI-seq assay as a genome-wide screen to identify proteins that interact with $\mathrm{H} 2 \mathrm{~A}$ or $\mathrm{H} 2 \mathrm{~A} . \mathrm{Z}$ using stable cell lines that expressed fusion proteins of the N-terminal part of GFP with $\mathrm{H} 2 \mathrm{~A}$ or H2A.Z. The fusion proteins were expressed at levels similar to or lower than the endogenous protein. Furthermore, the H2A.Z fusion protein was incorporated into chromatin in a similar manner to endogenous $\mathrm{H} 2 \mathrm{~A}$. $\mathrm{Z}$ and was able to rescue the H2A.Z knockdown. Thus, it is very likely that expression of these fusion proteins does not globally disrupt chromatin structure or gene expression in the cells. Using bPPI-seq, we identified 217 H2A.Z-interacting proteins and 295 H2A-interacting proteins. Our data indicated that the majority of the proteins interacting with $\mathrm{H} 2 \mathrm{~A}$ or $\mathrm{H} 2 \mathrm{~A} . \mathrm{Z}$ fall into different functional groups. While H2A.Z-interacting proteins are highly enriched for transcriptional regulation functions, such proteins are not enriched in the H2A-interacting set. This suggests that the functional differences between H2A and H2A.Z may be controlled by their interacting proteins.

Deposition of H2A.Z onto chromatin is DNA replication-independent and mediated by the ANP32E-contain- 
A Osr1 motif determined by SELEX

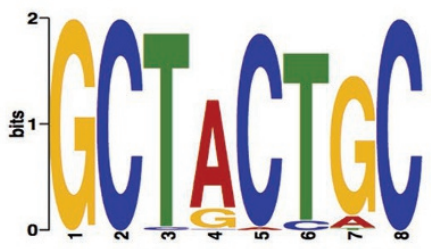

C

Fractions of $\mathrm{H} 2 \mathrm{~A} . \mathrm{Z}$ peaks with or without Osr1 motif

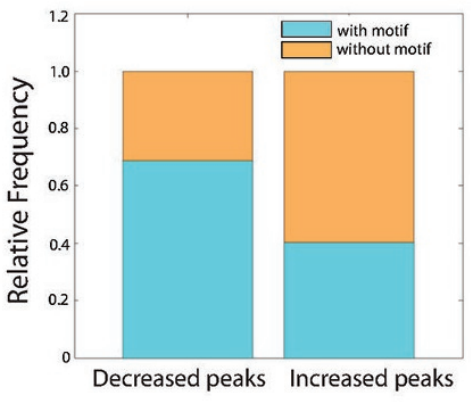

E Fractions of H2A.Z peaks with

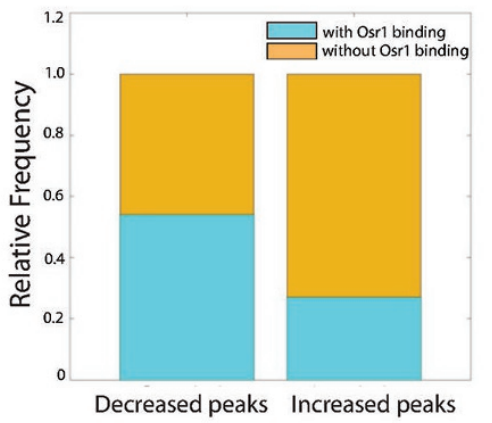

B

\section{by knocking down Osr1}

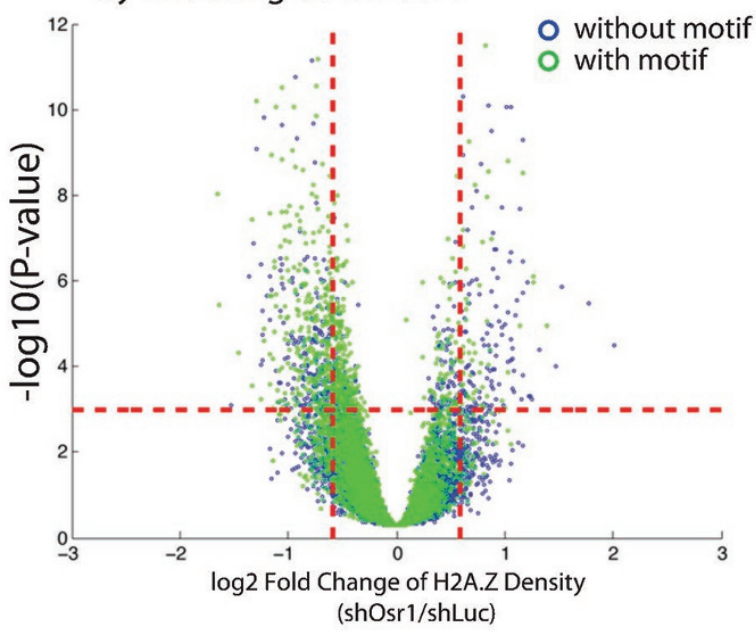

D

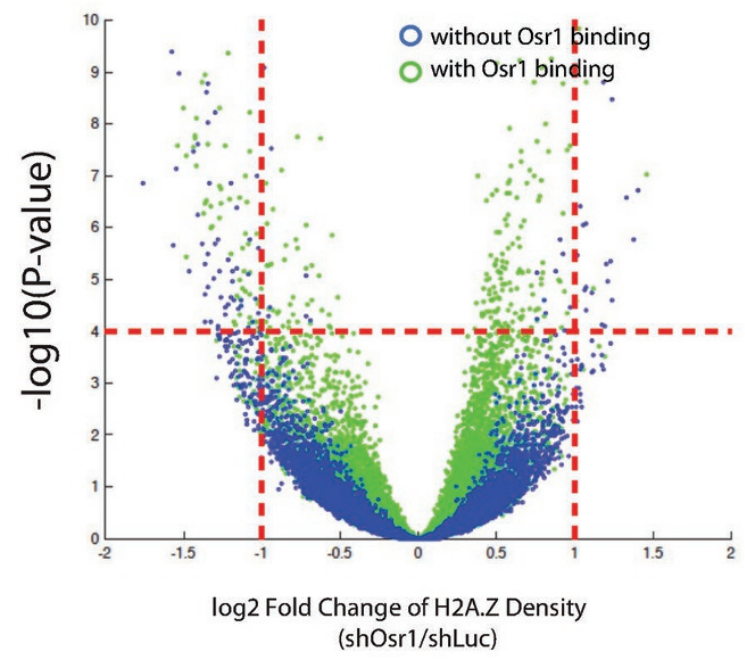

Figure 7 Osr1 facilitates incorporation of H2A.Z to regions containing target motifs. (A) The Osr1 motif logo identified by SELEX using bacterially expressed GST-Osr1 protein and mouse genomic DNA. (B) Volcano plot of changes in H2A.Z ChIPSeq peaks after knocking down Osr1. The blue and green circles indicate the H2A.Z peaks without and with an Osr1 motif, respectively. The vertical red lines indicate 1.5-fold change and horizontal red line indicates a significant $P$-value cutoff. (C) A significantly larger fraction of decreased H2A.Z peaks contain an Osr1 motif than the increased H2A.Z peaks. The fractions of the H2A.Z peaks containing Osr1 motif were calculated according to Supplementary information, Table S9. (D) Volcano plot of changes in H2A.Z ChIP-Seq peaks following knockdown of Osr1. The blue and green circles indicate the H2A.Z peaks without and with Osr1 binding, respectively. The vertical red lines indicate a two-fold change and horizontal red line indicates a significant $P$-value cutoff. (E) The observed relative frequency of decreased and increased H2A.Z peaks that have Osr1 binding. The frequency of the H2A.Z peaks that have Osr1 binding was calculated according to Supplementary information, Table S9.

ing SRCAP/TIP60 complexes [14, 16, 17, 24]. However, it is not clear how H2A.Z finds its highly specific target sites in the genome. While our data and those of others suggested that deposition of H2A.Z facilitates the binding of transcription factors to their target sites $[8,11]$, we hypothesized that H2A.Z's direct interaction with 
sequence-specific transcription factors may guide H2A.Z to specific regions in the genome. Indeed, we found that many sequence-specific DNA-binding transcription factors, including Osr1, directly interact with H2A.Z. Our results indicate that Osr1 directly interacts with $\mathrm{H} 2 \mathrm{~A}$. Z, co-localizes with H2A.Z on chromatin and contributes to the targeting of H2A.Z to a large number of sites containing the recognition motif of Osr1. The observation that H2A.Z and Osr1 appear to regulate a shared set of genes could be explained either by them functioning in the same pathway or by Osr1 expression requiring H2A.Z. We suggest that both mechanisms could contribute to the regulation of the shared target genes because H2A.Z targeting appears to be regulated by Osr1 in cells.

In this study, we randomly picked Osr1 to demonstrate the sensitivity and robustness of the relatively weak interactions detected by bPPI-seq. Since bPPI-seq revealed multiple transcription factors that interact with H2A.Z, it is highly likely that other transcription factors may also be involved in the recruitment of H2A.Z to target regions and it would be interesting to test this in future studies.

We have shown that bPPI-seq is an effective, rapid and inexpensive method for a genome-wide screen for proteins interacting with a bait protein under physiological conditions. Compared to the current affinity purification/mass spectrometry method, our bPPI-seq method has the following advantages: (1) the proteins detected by bPPI-seq are most likely direct interactors because of the requirement of spatial proximity to reconstitute active GFP; (2) bPPI-seq is capable of detecting weak and transient interactions that require a physiological environment and may not be detectible by other methods; (3) bPPI-seq detects protein interactions in vivo under physiological conditions and thus avoids in vitro artifacts; and (4) bPPI-seq reveals the location of potential interacting domains within a protein based on the fusion site. One caveat for using bPPI-seq is the necessity to control the expression of bait proteins; too high expression of bait proteins, such as by transient expression, may result in detection of artifactual interactions. Therefore, we cannot exclude the possibility that there may be some false positives using bPPI-seq due to its sensitivity. Thus other strategies such as co-localization on chromatin by ChIPSeq, if applicable, or co-IP may be needed to confirm a true interaction. Although here, we have only demonstrated the applicability of bPPI-seq to the identification of proteins interacting with the chromatin proteins $\mathrm{H} 2 \mathrm{~A}$ and H2A.Z, we fully expect the approach can be widely applied to identify interacting partners of proteins from other cellular compartments. Thus the method will be instrumental for understanding protein components and interaction networks in various cellular processes.

\section{Materials and Methods}

\section{Stable cell lines for bPPI-seq}

NIH-3T3 Tet-on cells were transfected with pTight-GFPN, pTight-GFPN-H2A or pTight-GFPN-H2A.Z. After $48 \mathrm{~h}$ transfection, we treated the cells with Hygromycin B $(200 \mu \mathrm{g} / \mathrm{ml})$ to select stably transfected cells. After 2 weeks, clones were picked in which the expression level of GFPN, GFPN-H2A or GFPN-H2A.Z was comparable to that of endogenous H2A or H2A.Z with $2 \mu \mathrm{g} /$ $\mathrm{ml}$ Doxycycline treatment for $48 \mathrm{~h}$.

\section{Cell lines, plasmids and antibodies}

NIH/3T3 Tet-on 3G cells (Clontech), GP2 293 cells (Clontech) and Lenti-X 293 T cells (Clontech) were routinely cultured in DMEM + GlutaMAX (Gibco) supplemented with 10\% FBS (Sigma). ReMTH-GFPC1/2/3 and Bait-GFPN-Hygro plasmids were provided by Drs Zhiyong Ding and Gordon Mills (M.D. Anderson Cancer Center) [36]. The pEF1a-Bait-GFPN-Hygro plasmid was constructed by replacing the CMV promoter of Bait-VNN-Hygro with the EF1a promoter from pEF1a-IRES-AcGFP1 (Clontech). cDNAs of mouse H2A and H2A.Z were subcloned in-frame with an HA tag into the pEF1a-GFP-N-Hygro Vector. The mouse H2B (HistH2bc), Osr1, Tinf2 and ANP32E cDNAs were subcloned inframe with a Flag tag and GFP-C into the pEF1a-Hygro Vector. The virus packaging vectors were $\mathrm{pEco}$ (Clontech) for retrovirus and pGreenPuro, ps-PAX2 and plp-VSVG for lentivirus (System Biosciences). pMal-c2 vector (New England Biolabs) and pGSTag vector (Addgene) were used for expression of MBP and GST fusion proteins.

The following antibodies were used in this study: anti-HA (Roche, 11867423001); anti-FLAG (Sigma, F1804); anti-HA-HRP (Santa Cruz, sc7392 HRP); anti-FLAG-HRP (Sigma, A8592); anti-Osr1 (Santa Cruz, sc68392); anti-H2A.Z (Abcam, ab4174).

\section{Screening for H2A.Z-interacting proteins}

ReMTH-VNC 1, 2 and 3 (three different reading frames) and pEco were co-transfected into GP2 293 cells using Lipofectamine 2000 (Invitrogen). Supernatants containing packaged viral particles were collected $48 \mathrm{~h}$ after transfection. About $30 \mathrm{ml}$ viral particles were used to infect $5 \times 10^{7} 3 \mathrm{~T} 3$ Tet-on $3 \mathrm{G}$ cells expressing GFPN-H2A.Z, GFPN-H2A or GFPN alone to avoid multiple integrations. $48 \mathrm{~h}$ post-infection, cells were selected in DMEM containing $2 \mu \mathrm{g} / \mathrm{ml}$ puromycin (Sigma) for 2 days and maintained in $1 \mu \mathrm{g} / \mathrm{ml}$ puromycin for 3 days. Then, an equal number of cells infected with viruses for the three reading frames were pooled and induced by $2 \mu \mathrm{g} / \mathrm{ml}$ Doxycycline for $48 \mathrm{~h}$ before sorting for green cells. GFP-positive cells were sorted with FACSAria (BD Biosciences).

\section{bPPI-seq library preparation and sequencing}

Total RNAs of the sorted cells were extracted using QIAzol (Qiagen) and purified with miRNeasy micro kit (Qiagen) and DNase set (Qiagen). Purified RNAs were reverse transcribed using PrimeScript Reverse Transcriptase (Takara) with a PolyT primer. The cDNAs were amplified by a GFPC-specific (VNC primer) and T7 primers, and fragmented to 200 to $500 \mathrm{bp}$ by sonication. The DNA ends were repaired using an End-It DNA End-repair Kit (Epicentre), followed by treatment with Klenow Fragment exo(New England Biolabs) to generate a protruding 3'A base used for 
adaptor ligation. Following ligation of a pair of top-Phops-oligo$17 \mathrm{bp} / \mathrm{T} 7 \mathrm{Mme} \mathrm{I}-18 \mathrm{bp}$ adaptors, fragments between 250 and 650 bp were isolated from agarose gel. Then the DNA was amplified by MmeI-SD-1, MmeI-SD-2, MmeI-SD-3 and T7 primers to add a MmeI recognition site at both ends, followed by digestion with MmeI (New England Biolabs). After treatment with Klenow Enzyme (New England Biolabs), indexed libraries were prepared with a Multiplexing Sample Preparation Oligonucleotide Kit (Illumina) according to the user's manual. The fragments between 250 and $500 \mathrm{bp}$ were isolated for sequencing on an Illumina next-generation sequencing platform. The primers used for library preparation are listed below:

PolyT primer: 5'-GCAAATACGACTCACTATAGGGATCCTTTTTTTTTTTTTTTT-3'

VNC primer: 5'-AAAGACCCCAACGAGAAGC-3'

T7 primer: 5'-GCAAATACGACTCACTATAGGGATC-3'

top-Phosp-oligo-17bp: 5'-GGTGGATGGATGATGTGAGAATATA-3'

T7MmeI-18bp: 5'-GCAAATACGACTCACTATAGGGATCTCCGACACATCATCCATCCACCT-3

MmeI-SD-1: 5'-CGTCCCAGACTTCCGACGTCGATATATCTGGAGAAAG-3'

MmeI-SD-2: 5'-GACTACGCAGGTCCGACATCGATATATCTGGAGAAAG-3'

MmeI-SD-3: 5'-CAGGTCGACCATCCGACGGCGATATATCTGGAGAAAG-3'

\section{Co-immunoprecipitation to detect interactions in mammali- an cells}

The HA-tagged H2A or H2A.Z constructs were co-transfected with one of the four constructs expressing FLAG-tagged H2B, ANP32E, Osr1 or Tinf2 protein into NIH3T3 Tet-on 3G cells. $48 \mathrm{~h}$ post-transfection, whole-cell lysates were prepared by washing the cells twice with PBS and lysed in ice-cold lysis buffer containing $20 \mathrm{mM}$ Tris-HCl pH 7.5, 1\% NP40, 1 mM EDTA, $150 \mathrm{mM} \mathrm{NaCl}$, $10 \%$ glycerol and proteinase inhibitor. Chromatin lysates were prepared as described [37]. Anti-FLAG and anti-HA antibody were conjugated with protein $\mathrm{G}$ beads (Invitrogen). The lysates were incubated with antibody-protein $\mathrm{G}$ beads with rotation at $4{ }^{\circ} \mathrm{C}$ overnight. The beads were then washed four times with lysis buffer containing $150-500 \mathrm{mM} \mathrm{NaCl}$ and $0.1 \% \mathrm{NP}-40$ or $1 \times$ buffer $\mathrm{D}$ [37]. The bound proteins were eluted from the beads by boiling in SDS sample buffer with $100 \mathrm{mM}$ DTT for $5 \mathrm{~min}$. For western blotting, samples were resolved on SDS-PAGE gels according to standard procedures.

\section{MBP-H2A.Z and GST-Osr1 pull-down assay}

The various constructs expressing fusion proteins between H2A.Z (full length), H2A.Z-N (amino acids 1-40), H2A.Z-N+M (amino acids 1-80), H2A.Z-M (amino acids 41-80), H2A.Z-M+C (amino acids 41-127), H2A.Z-C (amino acids 81-127) or H2A (full length) and maltose-binding protein (MBP) were made by inserting the corresponding cDNAs into pMal-C2 between Bam HI and HindIII sites. Constructs expressing fusion proteins between Osr1 (full length), Osr1-N (amino acids 1-133) or Osr1-C (amino acids 134-266) and GST with a FLAG tag at the C-terminus were made by inserting the corresponding cDNAs into pGSTag between $\mathrm{XbaI}$ and $X h o$ I sites. MBP and GST fusion proteins were expressed in BL21-Gold-DE3 cells (Stratagene). Briefly, cells transformed with the expression constructs were cultured in $3 \mathrm{ml}$ Luria Broth with $100 \mu \mathrm{g} / \mathrm{ml}$ carbenicilin (Invitrogen) at $37{ }^{\circ} \mathrm{C}$ overnight, which was diluted into $150 \mathrm{ml}$ Luria Broth containing $100 \mu \mathrm{g} / \mathrm{ml}$ carbenicilin and further incubated for $3 \mathrm{~h}$ at $37{ }^{\circ} \mathrm{C}$ with shaking. The expression of fusion proteins was induced by $1 \mathrm{mM}$ IPTG (Corning) for $2 \mathrm{~h}$. Cells were collected by low-speed centrifugation, re-suspended in $3 \mathrm{ml}$ binding buffer ( $20 \mathrm{mM}$ Tris- $\mathrm{HCl} \mathrm{pH} 7.5,150 \mathrm{mM} \mathrm{NaCl}$, $1 \mathrm{mM}$ EDTA, $1 \mathrm{mM}$ DTT, 0.1\% NP40, 5\% glycerol and proteinase inhibitor), and lysed by three cycles of freeze-and-thaw. Following sonication and high-speed centrifugation, the supernatant was mixed with Amylose Resin (New England Biolabs) for MBP fusion protein or glutathione-Sepharose $4 \mathrm{~B}$ beads (GE Healthcare) for GST fusion protein for $1 \mathrm{~h}$ at $4{ }^{\circ} \mathrm{C}$. The beads were washed four times with binding buffer. The bound proteins were eluted from one-half of the beads while keeping another half beads with proteins still bound in binding buffers. MBP fusion protein was eluted by $20 \mathrm{mM}$ Tris- $\mathrm{HCl} \mathrm{pH}$ 7.5, $200 \mathrm{mM} \mathrm{NaCl}, 1 \mathrm{mM}$ DTT and $10 \mathrm{mM}$ maltose. GST fusion protein was eluted by $50 \mathrm{mM}$ Tris$\mathrm{HCl} \mathrm{pH} 7.5$ and $10 \mathrm{mM}$ reduced glutathione. Then MBP or GST fusion protein was mixed with GST fusion protein-bound beads or MBP fusion protein-bound beads, respectively, and incubated with rotation overnight at $4{ }^{\circ} \mathrm{C}$. The beads were washed five times with binding buffer containing $150-500 \mathrm{mM} \mathrm{NaCl}$ and bound proteins were eluted with SDS sample buffer with $100 \mathrm{mM}$ DTT, and resolved by SDS-PAGE. Western blotting was performed as described above.

\section{GST-Osr1 genomic-SELEX}

GST-Osr1 fusion protein on sepharose beads was generated as described above. Mouse genomic DNA ( $1 \mathrm{~g})$ was sonicated in a Diagenode Bioruptor (level M, for a total of 10 min alternating 15 $\mathrm{s}$ on and $15 \mathrm{~s}$ off) to a size range of 100-400 bp. Genomic-SELEX was performed as described [34] with some modification. Briefly, washed unloaded sepharose beads and GST-Osr1 beads were incubated with gentle agitation for $30 \mathrm{~min}$ at $37^{\circ} \mathrm{C}$ in the presence of $500 \mathrm{ng}$ of sonicated DNA. The resin was washed five times with SELEX binding buffer (20 mM Tris- $\mathrm{HCl} \mathrm{pH}$ 8.0, 10\% glycerol, 1 $\mathrm{mM}$ DTT, $300 \mathrm{mM} \mathrm{NaCl})$. DNA and protein complex were eluted by elution buffer $(50 \mathrm{mM}$ Tris- $\mathrm{HCl}, \mathrm{pH} 7.5$ and $10 \mathrm{mM}$ reduced glutathione). Eluted DNA was purified by MinElute Reaction Cleanup Kit (Qiagen). The DNA ends were repaired and a protruding $3^{\prime}$ A generated as described above. Following ligation of a pair of Solexa adaptors to the repaired ends, 150 to $400 \mathrm{bp}$ fragments were recovered from agarose gel. Then DNA was amplified using the index primers for 18 cycles and the 150-400 bp fragments were isolated from agarose gel again. About $300 \mathrm{ng}$ of the resultant library DNA was added again to the new empty sepharose beads and GST-Osr1 beads, and the process was repeated four times. The libraries enriched at rounds two, three and four were sequenced using Illumina high-throughput sequencing.

\section{Knockdown of H2A.Z and Osr 1 by $\operatorname{shRNA}$}

The RNA interference construct targeting mouse Osrl was generated by inserting the target oligos into pGreenPuro Lentivector (System Biosciences) and the shH2A.Z construct was described previously [8]. The target gene sequences are listed below:

shLuc: 5'-GTGCGTTGTTAGTACTAATCCTATTT-3'

shOsr1: 5'-CAGATATATTCACTCCAAA-3'

shH2A.Z: 5'-CCTTATTATCTCAGGACTCTA-3' (This target 
sequence is located in the $3^{\prime}$ UTR of $H 2 A . Z$ so that the rescuing $H 2 A . Z$ cDNA is resistant to this shRNA.)

\section{Transfection for ChIP-Seq and RNA-Seq}

A wide concentration range of pTight-H2A.Z-GFPN was used for transfection $(0,0.75,1.5,3.0,6.0,12 \mathrm{~g}$ together with $12,11.25$, $10.5,9,6,0 \mu \mathrm{g}$ of control pTight-GFPN, respectively) into tet on $3 \mathrm{G}$ cells. About $48 \mathrm{~h}$ later $2 \mu \mathrm{g} / \mathrm{ml}$ Doxycycline was added to induce expression for $48 \mathrm{~h}$ before fixing cells for anti HA Chip-seq. The shLuc control or knockdown cells were also cross-linked with formaldehyde, and chromatin fractions were prepared by sonication. The ChIP-seq experiments were performed as described [4]. The RNA-Seq was performed as described [38].

\section{Data analysis}

bPPI-Seq data analysis We constructed three bPPI-Seq libraries, namely void GFPN, H2A-GFPN and H2A.Z-GFPN. The reads from each bPPI-Seq library were mapped to the mouse genome (mm9) using tophat [22]. The number of reads located on coding region of each gene was counted in each library. Interactions with the GFPN-fused-protein will lead to insertion of tet-responsive promoter and lead expression of the interacting genes. Selected potentially interacting proteins were required to satisfy three criteria. (1) Genes should have moderate mRNA expression (RPKM >6) quantified by the RNA-seq data. (2) They should have more than six counts per million mapped reads from the bPPI-seq data. (3) The standard deviation of two replicates of the H2A.Z or H2A bPPI-seq (in RPKM) should be $\leq 4$. Among these potential interacting proteins, a protein was considered to interact with $\mathrm{H} 2 \mathrm{~A}$ or H2A.Z if the difference of read density of the gene was significant as compared to the GFPN control library, using the method developed by Audic and Claverie [23] (fasle-positive discover rate $<0.000001$; fold-change of read densities over eight-fold).

Gene ontology analysis H2A.Z- and H2A-specific interacting proteins are obtained by filtering out the overlap between the two sets of overlapping interacting proteins. We performed gene ontology analysis for the two sets of interacting proteins using the category of biological process in Gene Ontology Consortium [31].

ChIP-seq data analysis Raw sequence reads were aligned to the reference mouse genome (UCSC, mm9) by Bowtie [39]. We allowed for one read per position matching and removed redundant reads for each ChIP-seq library for the alignment. The enriched regions of $\mathrm{H} 2 \mathrm{~A} . \mathrm{Z}$ binding were identified using SICER (window sizes $=200 \mathrm{bp}$, gap $=200 \mathrm{bp}$, E-value $<0.001$, score $\geq 100)$ [40]. The enriched regions of Osr1 binding were identified using model-based analysis for ChIP-Seq (MACS) with $P$-value cutoff equal to $1 \mathrm{e}-5$ [40].

RNA-seq data analysis We identified 22490 uniquely known genes from the mouse genome ( $\mathrm{mm} 9$ ) using the UCSC genome browser database [41]. To quantify the mRNA level of genes, the RKPM (reads per kilobase of transcript per million mapped reads) values were computed over gene exons from the RNA-seq data sets. Differentially expressed genes between treated and control cells were identified by edgeR [42] using two replicates in each condition (FDR $<0.01$ and fold-change $>1.5$ or $<2 / 3$ ).
Correlation analysis between two experiments We quantified the similarity between the $\log _{2}$-fold changes (knockdown over control) for the two knockdown experiments (shH2A.Z and shOsr1) for both H2A.Z ChIP-seq data sets and RNA-seq data sets. For the H2A.Z ChIP-seq, all identified peaks from shLuc, shOsr1 and shH2A.Z experiments in one of the replicates were considered, and a set of $N$ combined peaks was obtained by combining the identified peaks if they had any portions of overlap. We calculated the $\log _{2}$-fold changes of read density (normalized by library size) of H2A.Z binding in knockdown experiments versus control for all the $N$ combined peak regions. The Pearson correlation coefficient was computed between the $\log _{2}$-fold changes of the two knockdown experiments to quantify their similarity. The same calculation was applied to the RNA-seq data sets, but the gene exon regions were considered instead of the combined peak regions and the RPKM measure was used instead of the read density measure. We also applied the same strategy to examine the reproducibility between two RNA-seq replicates, but we computed the correlation between the $\log _{2}$ RPKM measure of the two replicates instead of the $\log _{2}$-fold change. Note that combined peak regions (in ChIP-seq analysis) or genes (in RNA-seq analysis) were excluded if the $\log _{2}$ of the total read counts per million (CPM) from both replicates of the knockdown experiment and control experiment in the regions was less than 3 .

\section{Motif analysis}

To find the Osr1 binding motifs, we applied a motif search algorithm called MEME-ChIP [43] to the target genomic sequences identified by the GST-Osr1 SELEX assay.

The raw sequence reads from GST-Osr1 SELEX were aligned to the mouse genome (mm9) by Bowtie, and 144 Osr1-enriched regions were identified using MACS. Among these regions, 67 peaks were selected based on their $P$-values. In particular, a region size equalling $200 \mathrm{bp}$ was considered for each of the peaks, and each region was located +100 and -100 bp around the peak summit defined by MACS. By applying MEME-ChIP to the sequences in these selected regions and requiring the maximum width of the searching motif to be eight, we found a motif GCTACTGC (E-value $=1.2 \mathrm{e}-72$ ) as shown in Figure 7A. The motif we found was highly consistent with the Osr1 binding motif discovered by others [35].

\section{Analysis of differential H2A.Z binding between shOsrl and shLuc}

We quantitatively compared two H2A.Z ChIP-seq data sets from shOsr1 and shLuc cells in order to identify differential H2A.Z binding sites (peaks) between the two samples. In particular, we employed a normalization method to the two samples, in which the scale factor for normalizing the sequencing depth was computed based on the average of the read densities in the ChIP-enriched regions. Compared to the traditional sequencing depth normalization method [44] (i.e., read counts are normalized by the number of all mapped reads), our normalization method was probably more suitable to the ChIP-seq data sets in which a real biological global decrease/increase of the total number of reads occurred in the knockdown samples.

Prior to the computation of differential peaks, the sequencing data were required to go through several pre-processed steps described as follows:

(1) MACS/SICER was used to find out the coordinates of the 
ChIP-enriched regions (Peaks) for both samples.

(2) A unified set of peaks was obtained from the two samples by taking the union of their peaks found in step 1 and combining any two peaks if they are overlapped. A peak in the unified set was considered to be a "common" peak if it overlapped with at least one peak in both samples.

(3) The number of reads $\left(R_{K}\right.$ and $\left.R_{C}\right)$ in each unified peak region was computed for both samples, where the subscript $K$ and $C$ correspond to the knockdown and control samples, respectively.

(4) The read density was computed for each peak $\left(r_{K}\right.$ and $\left.r_{C}\right)$, corresponding to the number of read counts divided by the length of the peak.

(5) The values $M$ and $A$ were calculated for each peak $i$ using the following equations:

$$
\text { and } \quad \begin{aligned}
M^{i} & =\log _{2}\left(r_{k}^{i} / r_{C}{ }^{i}\right) \\
A^{i} & =\frac{1}{2}\left(\log _{2}\left(r_{k}^{i}\right)+\log _{2}\left(r_{C}^{i}\right)\right),
\end{aligned}
$$

where $r_{k}^{i}$ and $r_{C}^{i}$ are the read density of the peak $i$.

Next we compared the read densities in the knockdown and control samples. To normalize the read densities and remove any systematic bias between the two samples, we assumed that the average of the read densities of the most ChIP-enriched regions was conserved. This assumption was analogous to the assumption that the average of the activities of the house keeping genes is conserved.

In our analysis, we focused on the set of common peaks defined in the data pre-processing step 2. About 1000 common peaks with the largest value of $A$ were selected. Using the above assumption, a scale factor $S$ was calculated to make the average of the read density in the 1000 peaks to be the same, where $S=\left\langle r_{k}\right\rangle \mid\left\langle r_{\mathrm{C}}\right\rangle$, and $\left\langle r_{k}>\right.$ or $\left\langle r_{\mathrm{C}}>\right.$ is the average of the read densities in the 1000 common peaks of knockdown and control samples, respectively. The new read densities of the knockdown sample were equal to $r_{k}^{i}$ divided by $S$.

For each peak in the unified set, we calculated a $P$-value to quantify the significance of the differential H2A.Z binding using the method developed by Audic and Claverie [23]. In particular, the values of normalized read counts were used as inputs in the method [23], which was equal to $\left(r_{k}^{i} / S\right)$ multiply the width of the region. A peak was determined to be a significant differential binding peak if $P$-value $<0.001,|\mathrm{M}|>\log _{2}(1.5)$, and $\mathrm{A}>25$. A volcano plot of $-\log _{10}(P$-value $)$ versus $M$ was shown in Figure $7 \mathrm{~B}$ for peaks with $\mathrm{A}>25$.

Dependency of Osr1 motif and the directions of H2A.Z differential peaks

To elucidate the relationship between Osr1 and H2A.Z binding, we examined whether the directions of the differential H2A.Z binding regions were dependent on the existence of an Osr1 binding motif within the regions. In our analysis, we used FIMO [45], a TFBS motif identification software, to find the occurrence of the Osr1 binding motif within the differential H2A.Z bindings regions. The occurrence is considered to be significant with a $P$-value $<$ 0.0001 .

The result was shown in a $2 \times 2$ contingency table (Supplementary information, Table S10), which has a very significant $P$-value that equals to 0.0 (calculated by $\chi^{2}$-test). Therefore, as shown by the contingency table, the direction of differential H2A.Z bindings is strongly dependent on the existence of an Osr1 binding motif within the binding region. The relative frequency for each element in the contingency table was computed in which each element was divided by the sum of its row. A result of the relative frequencies was plotted in Figure 7C, which showed that H2A.Z binding was more likely to be decreased in regions containing the Osr1 binding motif when Osr1 was knocked down.

\section{Data accession}

The sequencing data have been deposited in GEO (GSE80425).

\section{Acknowledgments}

We thank the NHLBI DNA Sequencing Core facility for sequencing the libraries and the NHLBI Flow Cytometry Core facility for sorting the cells. The work was supported by Division of Intramural Research, NHLBI, NIH. BN was supported by the National Key Research and Development Project (2016YFA0502203). YZ was supported by a PhD Student Fellowship from the China State Scholarship Fund (2013-2014).

\section{Author Contributions}

$\mathrm{KZ}$ conceived the project. YZ performed the experiments. WLK analyzed the data. SL, KC, WJ, QT and WL contributed to the experiments or data analysis. $\mathrm{BN}$ and $\mathrm{KZ}$ directed the project. YZ, WLK and KZ wrote the manuscript.

\section{Competing Financial Interests}

The authors declare no competing financial interests.

\section{References}

1 Larochelle M, Gaudreau L. H2A.Z has a function reminiscent of an activator required for preferential binding to intergenic DNA. EMBO J 2003; 22:4512-4522.

2 Faast R, Thonglairoam V, Schulz TC, et al. Histone variant H2A.Z is required for early mammalian development. Curr Biol 2001; 11:1183-1187.

3 Clarkson MJ, Wells JR, Gibson F, Saint R, Tremethick DJ. Regions of variant histone His2AvD required for Drosophila development. Nature 1999; 399:694-697.

4 Barski A, Cuddapah S, Cui K, et al. High-resolution profiling of histone methylations in the human genome. Cell 2007; 129:823-837.

5 Zhang H, Roberts DN, Cairns BR. Genome-wide dynamics of Htz1, a histone H2A variant that poises repressed/basal promoters for activation through histone loss. Cell 2005; 123:219-231.

6 Weber CM, Henikoff JG, Henikoff S. H2A.Z nucleosomes enriched over active genes are homotypic. Nat Struct Mol Biol 2010; 17:1500-1507.

$7 \mathrm{Ku}$ M, Jaffe JD, Koche RP, et al. H2A.Z landscapes and dual modifications in pluripotent and multipotent stem cells underlie complex genome regulatory functions. Genome Biol 2012; 13:R85.

$8 \mathrm{Hu}$ G, Cui K, Northrup D, et al. H2A.Z facilitates access of active and repressive complexes to chromatin in embryonic stem cell self-renewal and differentiation. Cell Stem Cell 
2013; 12:180-192.

9 SubramanianV, Fields PA, Boyer LA. H2A.Z: a molecular rheostat for transcriptional control. F1000Prime Rep 2015; 7:01.

10 Marques M, Laflamme L, Gervais AL, Gaudreau L. Reconciling the positive and negative roles of histone H2A.Z in gene transcription. Epigenetics 2010; 5:267-272.

11 Li Z, Gadue P, Chen K, et al. Foxa2 and H2A.Z mediate nucleosome depletion during embryonic stem cell differentiation. Cell 2012; 151:1608-1616.

12 Skene PJ, Henikoff S. Histone variants in pluripotency and disease. Development 2013. 140:2513-2524.

13 Suto RK, Clarkson MJ, Tremethick DJ, Luger K. Crystal structure of a nucleosome core particle containing the variant histone H2A.Z. Nat Struct Biol 2000; 7:1121-1124.

14 Choi J, Heo K, An W. Cooperative action of TIP48 and TIP49 in H2A.Z exchange catalyzed by acetylation of nucleosomal H2A. Nucleic Acids Res 2009; 37:5993-6007.

15 Vardabasso C, Gaspar-Maia A, Hasson D, et al. Histone variant H2A.Z.2 mediates proliferation and drug sensitivity of malignant melanoma. Mol Cell 2015; 59:75-88.

16 Obri A, Ouararhni K, Papin C, et al. ANP32E is a histone chaperone that removes H2A.Z from chromatin. Nature 2014; 505:648-653.

17 Mao Z, Pan L, Wang W, et al. Anp32e, a higher eukaryotic histone chaperone directs preferential recognition for H2A.Z. Cell Res 2014; 24:389-399.

18 Alatwi HE, Downs JA. Removal of H2A.Z by INO80 promotes homologous recombination. EMBO Rep 2015; 16:986994.

$19 \mathrm{Hu}$ CD, Chinenov Y, Kerppola TK. Visualization of interactions among bZIP and Rel family proteins in living cells using bimolecular fluorescence complementation. Mol Cell 2002; 9:789-798.

20 Ding Z, Liang J, Lu Y, et al. A retrovirus-based protein complementation assay screen reveals functional AKT1-binding partners. Proc Natl Acad Sci USA 2006; 103:15014-15019.

21 Liu D, Yang X, Yang D, Songyang Z. Genetic screens in mammalian cells by enhanced retroviral mutagens. Oncogene 2000; 19:5964-5972.

22 Trapnell C, Pachter L, Salzberg SL. TopHat: discovering splice junctions with RNA-Seq. Bioinformatics 2009; 25:1105-1111.

23 Audic S, Claverie JM. The significance of digital gene expression profiles. Genome Res 1997; 7:986-995.

24 Ruhl DD, Jin J, Cai Y, et al. Purification of a human SRCAP complex that remodels chromatin by incorporating the histone variant H2A.Z into nucleosomes. Biochemistry 2006; 45:5671-5677.

25 Kobor MS, Venkatasubrahmanyam S, Meneghini MD, et al. A protein complex containing the conserved Swi2/Snf2-related ATPase Swr1p deposits histone variant H2A.Z into euchromatin. PLoS Biol 2004; 2:E131.

26 Krogan NJ, Keogh MC, Datta N, et al. A Snf2 family ATPase complex required for recruitment of the histone $\mathrm{H} 2 \mathrm{~A}$ variant Htz1. Mol Cell 2003; 12:1565-1576.

27 Mizuguchi G, Shen X, Landry J, Wu WH, Sen S, Wu C. ATP-driven exchange of histone $\mathrm{H} 2 \mathrm{AZ}$ variant catalyzed by SWR1 chromatin remodeling complex. Science 2004. 303:343-348.
28 Ranjan A, Mizuguchi G, FitzGerald PC, et al. Nucleosome-free region dominates histone acetylation in targeting SWR1 to promoters for H2A.Z replacement. Cell 2013; 154:1232-1245.

$29 \mathrm{Wu} \mathrm{WH}$, Alami S, Luk E, et al. Swc2 is a widely conserved H2AZ-binding module essential for ATP-dependent histone exchange. Nat Struct Mol Biol 2005; 12:1064-1071.

30 Yen K, Vinayachandran V, Pugh BF. SWR-C and INO80 chromatin remodelers recognize nucleosome-free regions near +1 nucleosomes. Cell 2013; 154:1246-56.

31 Huang da W, Sherman BT, Lempicki RA. Systematic and integrative analysis of large gene lists using DAVID bioinformatics resources. Nat Protoc 2009; 4:44-57.

32 Jensen K, Santisteban MS, Urekar C, Smith MM. Histone H2A.Z acid patch residues required for deposition and function. Mol Genet Genomics 2011; 285:287-296.

33 Subramanian V, Mazumder A, Surface LE, et al. H2A.Z acidic patch couples chromatin dynamics to regulation of gene expression programs during ESC differentiation. PLoS Genet 2013; 9:e1003725.

34 Reiss DJ, Mobley HL. Determination of target sequence bound by PapX, repressor of bacterial motility, in flhD promoter using systematic evolution of ligands by exponential enrichment (SELEX) and high throughput sequencing. J Biol Chem 2011; 286:44726-38.

35 Badis G, Berger MF, Philippakis AA, et al. Diversity and complexity in DNA recognition by transcription factors. Science 2009; 324:1720-1723.

36 Ding Z, Liang J, Lu Y, et al. A retrovirus-based protein complementation assay screen reveals functional AKT1-binding partners. Proc Natl Acad Sci USA 2006; 103:15014-15019.

37 Draker R, Ng MK, Sarcinella E, Ignatchenko V, Kislinger T, Cheung P. A combination of H2A.Z and H4 acetylation recruits $\mathrm{Brd} 2$ to chromatin during transcriptional activation. PLoS Genet 2012; 8:e1003047.

38 Wei G, Hu G, Cui K, Zhao K. Genome-wide mapping of nucleosome occupancy, histone modifications, and gene expression using next-generation sequencing technology. Methods Enzymol 2012; 513:297-313.

39 Langmead B, Trapnell C, Pop M, Salzberg SL. Ultrafast and memory-efficient alignment of short DNA sequences to the human genome. Genome Biol 2009; 10:R25.

40 Zhang Y, Liu T, Meyer CA, et al. Model-based analysis of ChIP-Seq (MACS). Genome Biol 2008; 9:R137.

41 Karolchik D, Hinrichs AS, Furey TS, et al. The UCSC Table Browser data retrieval tool. Nucleic Acids Res 2004; 32(Database issue):D493-D496.

42 Robinson MD, McCarthy DJ, Smyth GK. edgeR: a Bioconductor package for differential expression analysis of digital gene expression data. Bioinformatics 2010; 26:139-140.

43 Machanick P, Bailey TL. MEME-ChIP: motif analysis of large DNA datasets. Bioinformatics 2011; 27:1696-1697.

44 Kharchenko PV, Tolstorukov MY, Park PJ. Design and analysis of ChIP-seq experiments for DNA-binding proteins. Nat Biotechnol 2008; 26:1351-1359.

45 Grant CE, Bailey TL, Noble WS. FIMO: scanning for occurrences of a given motif. Bioinformatics 2011; 27:1017-1018.

(Supplementary information is linked to the online version of the paper on the Cell Research website.) 